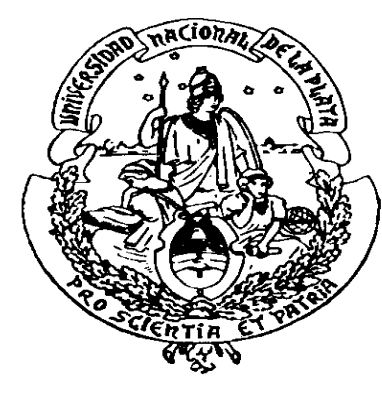

Universidad Nacional de La Plata

Facultad de Ciencias Exactas

Departamento de Matemática

\title{
SOBRE LOS GRAFOS VPT Y LOS GRAFOS EPT
}

\author{
Autor: \\ María Pía Mazzoleni \\ Director: \\ Dra. Marisa Gutierrez \\ $\underline{\text { Co-Director: }}$ \\ Dra. Liliana Alcón \\ Tesis para obtener el título de
}

DOCTOR DE LA FACULTAD DE CIENCIAS EXACTAS, AREA MATEMÁTICA 
A mis padres Susana y Alfredo, mi hermana Agus, mis abuelos Lila y Ruben, y mi abuela de corazón Margot. 


\section{Agradecimientos}

A mis papás, Susana y Alfredo. Por ser mis principales apoyos, por el esfuerzo que hicieron para ayudarme a cumplir mis objetivos.

A mi hermana, Agus. Por ser mi compañera incondicional. Por todos los momentos compartidos y por escucharme siempre.

A mi novio, Mariano. Por estar a mi lado durante todos estos años y acompañarme en cada momento.

A Vale, por tantos años de amistad, por mostrarse siempre interesada en mis cosas y estar siempre que lo necesité.

A Marisa y Liliana por haberme guiado en el desarrollo de este trabajo, por el aporte de su experiencia y conocimiento. Por haberme brindado su tiempo y también su confianza. Por su calidad humana.

A CONICET, por haberme provisto de una Beca de Posgrado sin la cual este trabajo no hubiese sido posible.

Al Departamento de Matemática de la Facultad de Ciencias Exactas de la Universidad Nacional de La Plata, por haberme permitido desarrollar las tareas correspondientes a este trabajo. 


\section{Índice general}

1. Introducción 1

2. Preliminares 7

3. Reconocimiento de los grafos vértice-interseción de caminos en árboles de grado acotado $\quad 14$

3.1. Resultados básicos . . . . . . . . . . . . . . . . . 15

3.2. Caracterización de $[\mathrm{h}, 2,1]$, para $h \geq 3 \ldots \ldots \ldots$

3.3. Complejidad . . . . . . . . . . . . . . . . 25

3.3.1. Una subclase que es reconocible en tiempo polinomial . 28

4. Caracterización de los grafos $[h, 2,1]$ por subgrafos inducidos prohibidos minimales 33

4.1. Preliminaries . . . . . . . . . . . . . . . . . 34

4.2. Condiciones necesarias para grafos VPT que son minimales no- $[\mathrm{h}, 2,1] \ldots \ldots \ldots \ldots \ldots \ldots \ldots$

4.3. Construyendo grafos minimales no- $[\mathrm{h}, 2,1] \ldots \ldots$. . . . . . 46

4.4. Caracterización de los grafos minimales no- $[\mathrm{h}, 2,1] \ldots \ldots$ 
5. Una condición necesaria para grafos EPT y una nueva familia de subgrafos inducidos prohibidos minimales $\quad 50$

5.1. Resultados básicos sobre cliques en grafos EPT . . . . . . . 52

5.2. Una condición necesaria para grafos EPT . . . . . . . . . . 54

5.3. Nuevos subgrafos prohibidos minimales para la clase EPT . . 56

6. Grafos EPT en árboles de grado acotado 61

6.1. Resultados conocidos . . . . . . . . . . . . . . 61

6.2. Nuestros resultados ................. 63

7. Conclusiones y trabajo futuro 68

$\begin{array}{ll}\text { Bibliografía } & 70\end{array}$ 


\section{Capítulo 1}

\section{Introducción}

El grafo de intersección de una familia de conjuntos es un grafo cuyos vértices son los miembros de la familia y la adyacencia es definida por la intersección no vacía de los correspondientes miembros. Los grafos de intersección son bastante conocidos y muy estudiados. Un trabajo dedicado a las clases de grafos de intersección es [30], donde se puede encontrar una gran colección de resultados.

Algunas clases de grafos definidas como intersección son hereditarias y pueden ser caracterizadas por subgrafos inducidos prohibidos minimales. Los elementos de las familias y las propiedades que las definen aparecen en varios contextos, modelando diferentes situaciones, inclusive de la vida real [30], lo que es un incentivo adicional para el estudio de estas clases.

Ejemplos clásicos son los grafos de intervalos y los grafos cordales.

Un grafo de intervalos es el grafo de intersección de una familia de intervalos en la recta real, o, en forma equivalente, el grafo vértice intersección de una familia de subcaminos de un camino. Llamamos Intervalos a la clase 
formada por los grafos de intervalos. Un grafo cordal es un grafo sin ciclos inducidos de longitud al menos cuatro. Llamamos Cordal a la clase formada por los grafos cordales. Gavril probó que un grafo es cordal si y sólo si es el grafo vértice intersección de una familia de subárboles de un árbol [13]. Ambas clases han sido cuidadosamente estudiadas en la literarura. En [7] encontramos un resumen sobre las diferentes clases de grafos.

Con el fin de definir nuevas clases de grafos representadas por subárboles, se imponen condiciones en los árboles, subárboles y en el tamaño de la intersección $[25,26]$.

Sean $h, s$ y $t$ enteros positivos; una $(\mathbf{h}, \mathbf{s}, \mathbf{t})$-representación de un grafo $G$ consiste de un árbol huésped $T$ y una colección $\left(T_{v}\right)_{v \in V(G)}$ de subárboles de $T$, tal que:

1. el grado máximo de $T$ es a lo sumo $h$;

2. todo subárbol $T_{v}$ tiene grado máximo a lo sumo $s$;

3. dos vértices $v$ y $v^{\prime}$ son adyacentes en $G$ si y sólo si los correspondientes subárboles $T_{v}$ y $T_{v^{\prime}}$ tienen al menos $t$ vértices en común en $T$.

La clase de grafos que tiene una $(h, s, t)$-representación es denotada $[\mathbf{h}, \mathbf{s}, \mathbf{t}]$.

Cuando no hay restricción en el grado máximo de $T$ o en el grado máximo de los subárboles, usamos $h=\infty$ y $s=\infty$ respectivamente. De ahí que, $[\infty, \infty, 1]=$ Cordal y $[2,2,1]=$ Intervalos. Las clases $[\infty, 2,1]$ y $[\infty, 2,2]$ son llamadas VPT (vertex intersection graph of paths in a tree) y EPT (edge intersection graph of paths in a tree) respectivamente en [15]; y UV y UE, respectivamente en [31]. 
VPT y EPT son clases incomparables de grafos, pues por el ejempplo el grafo $A_{4}$ dado en la Figura 3.6 pertenece a VPT-EPT y el ciclo inducido $C_{4}$ pertenece a EPT-VPT. Sin embargo, cuando el grado máximo del árbol huésped es tres la clase de los grafos VPT coincide con la clase de los grafos EPT.

Los grafos VPT son útiles en muchas áreas, entre las cuales cabe destacar la genética, arqueología y ecología.

Los grafos EPT son usados en aplicaciones de redes, donde el problema de planificación de llamadas no dirigidas en una red que es un árbol es equivalente al problema de colorear un grafo EPT. La red de comunicación está representada como un grafo no dirigido de interconexión, donde cada arista es asociada con una conexión física entre nodos. Una llamada no dirigida es un camino en la red. Cuando la red es un árbol, este modelo es claramente una representación EPT. Colorear el grafo EPT de forma tal que dos vértices adyacentes tengan distintos colores, significa que llamadas no dirigidas que comparten una conexión física tienen que planificarse en distintos momentos.

En los últimos años, el estudio de las clases $[h, s, t]$ ha merecido varias publicaciones en la literatura. En [12], el mínimo $t$ tal que un grafo dado pertenece a $[3,3, t]$ es estudiado. En [32], se muestra que $[3,3,1]=$ Cordal. En [21], los $[4,4,2]$ grafos son caracterizados y se da un algoritmo polinomial para su reconocimiento. En [20] y [11] respectivamente, las clases $[4,2,2]$ y $[4,3,2]$ son estudiadas. Reconocimiento, coloración y algunos otros problemas clásicos en la clase $[\infty, 2, t]$ son tratados en [18]. La relación entre diferentes clases de grafos de intersección de caminos en un árbol es analizada en [19]. 
En [14, 33], se muestra que el problema de reconocer a los grafos VPT es polinomial. Por otro lado, el reconocimiento de los grafos EPT es un problema NP-completo [15].

Esta Tesis está organizada de la siguiente forma:

El Capítulo 2 contiene definiciones que serán utilizadas en los capítulos siguientes y que son necesarias para entender el texto.

En el Capítulo 3 nos enfocamos en las clases $[h, 2,1]$ para cualquier $h \geq 3$ fijo; éstas son todas subclases de VPT. Caracterizamos a los grafos $[h, 2,1]$ usando el número cromático. Mostramos que el problema de decidir si un grafo VPT dado pertence a $[h, 2,1]$ es NP-completo, mientras que el problema de decidir si el grafo dado pertenece a $[h, 2,1]-[h-1,2,1]$ es NP-difícil. Ambos problemas permanecen difíciles aún cuando nos restringimos a la clase VPT $\cap$ Split. Adicionalmente, presentamos una subclase no trivial de VPT $\cap$ Split en la cual estos problemas son polinomiales. El caso $h=2$ no es considerado porque $[2,2,1]=$ Intervalos. Nuestros resultados se aplican para cualquier $h \geq 3$ fijo, pueden ser vistos como una generalización del caso $h=3$ el cual coincide con la clase $[3,2,1]=[3,2,2]=\mathrm{VPT} \cap \mathrm{EPT}=\mathrm{EPT}$ $\cap$ Cordal considerada en $[15,35]$.

Las clases $[h, 2,1]$, con $h \geq 2$, son cerradas por subgrafos inducidos, de ahí que cada una puede ser caracterizada por una familia de subgrafos inducidos prohibidos minimales. Tal familia es conocida sólo para $h=2$ [28] y hay algunos resultados parciales para $h=3$ [9]. En este Capítulo asociamos los subgrafos inducidos prohibidos minimales para $[h, 2,1]$ que son VPT con los grafos (color) críticos. Describimos cómo obtener subgrafos inducidos prohibidos minimales a partir de los grafos críticos, más aún, mostramos que 
la familia de grafos obtenida usando nuestro procedimiento es exactamente la familia de subgrafos inducidos prohibidos minimales para $[h, 2,1]$ que son VPT. Esta familia junto con la familia de subgrafos inducidos prohibidos minimales para VPT [24, 29], es la familia de subgrafos inducidos prohibidos minimales para $[h, 2,1]$, con $h \geq 3$.

En el Capítulo 4 caracterizamos la clase $[h, 2,1]$ por subgrafos inducidos prohibidos minimales para cada $h \geq 3$ fijo. Cabe destacar que, tomando $h=3$, obtenemos una caracterización por subgrafos inducidos prohibidos minimales para la clase VPT $\cap \mathrm{EPT}=\mathrm{EPT} \cap$ Cordal $=[3,2,2]=[3,2,1]$ (ver $[15])$.

En el Capítulo 5 damos una nueva condición necesaria para ser un grafo EPT. Para esto nos basamos en la estructura de los cliques de un grafo EPT. Además, encontramos una nueva familia de subgrafos inducidos prohibidos minimales para la clase EPT.

En el Capítulo 6 nos enfocamos en los grafos EPT que pueden ser representados en un árbol con grado acotado. Respondemos negativamente una pregunta que Golumbic, Lypshteyn y Stern dejaron abierta en [19], basándonos en la representación EPT que tienen los ciclos de un grafo EPT.

Finalmente, en el Capítulo 7, damos algunas conclusiones y analizamos cuáles son los trabajos futuros que nos gustaría realizar. 


\begin{tabular}{|c|c|c|}
\hline $\mathrm{h}$ & {$[\mathrm{h}, 2,1]$} & {$[\mathrm{h}, 2,2]$} \\
\hline 1 & -- & -- \\
\hline 2 & $\mathrm{P}$ & $\mathrm{P}$ \\
\hline 3 & $\mathrm{NP}$ & $\mathrm{NP}$ \\
\hline 4 & $\mathrm{NP}$ & $?$ \\
\hline$\vdots$ & $\vdots$ & $\vdots$ \\
$\vdots$ & $\vdots$ & $\vdots$ \\
$\vdots$ & $\vdots$ & $\vdots$ \\
\hline$\infty$ & $\mathrm{P}$ & $\mathrm{NP}$ \\
\hline
\end{tabular}

Figura 1.1: Esta tabla muestra cuál es la complejidad de reconocer a los grafos que pertenecen a las clases $[h, 2,1]$ y $[h, 2,2]$ para cualquier $h \geq 2$ fijo. 


\section{Capítulo 2}

\section{Preliminares}

Muchas de las definiciones y notaciones adoptadas en este trabajo son consideradas estándar en Teoría de Grafos, y serán utilizadas sin ninguna introducción previa. En general, tales definiciones pueden ser encontradas en los libros de J. Bondy y U. Murty y de T. McKee y F. McMorris [6, 30]. Las definiciones y notaciones que no son consideradas estándar son introducidas en cada capítulo. Es decir, cada capítulo tiene una pequeña introducción del asunto específico al que se refiere.

Un grafo es un par ordenado $G=(V, E)$ donde $V$ es un conjunto finito no vacío de vértices y $E$ es un conjunto de pares ordenados de vértices distintos llamados aristas. Si $G$ es un grafo, $V(G)$ y $E(G)$ denotan el conjunto de vértices y de aristas, respectivamente. $\operatorname{Si}|V(G)|=1, G$ es llamado trivial. Una arista $e=u v$ es incidente en los vértices $u$ y $v$, que son los extremos de $e$. Un vértice $u$ es adyacente a un vértice $v$ en $G$ si $u$ y $v$ son extremos de una misma arista de $G$. En este caso también decimos que $u$ y $v$ son vecinos en $G$. Dos aristas son adyacentes cuando tienen algún extremo en común. 
El vecindario abierto de un vértice $v$, representado por $N_{G}(v)$, es el conjunto de vértices adyacentes a $v$. El vecindario cerrado $N_{G}[v]$ es $N_{G}(v) \cup\{v\}$. Un vértice $v$ es aislado cuando $N(v)=\emptyset \mathrm{y}$ es universal cuando $N(v)=V(G)-\{v\}$. El grado de $v$, denotado por $d_{G}(v)$, es el cardinal de $N_{G}(v)$. Para simplificar, cuando no haya confusión, omitimos el subíndice $G$ y escribimos $N(v), N[v]$ o $d(v)$. Los vértices $u, v \in V(G)$ son llamados gemelos verdaderos si $N[u]=N[v]$.

Dos grafos $G$ y $H$ son isomorfos si existe una biyección $\psi$ de $V(G)$ en $V(H)$ tal que la arista $u v \in E(G)$ si y sólo si $\psi(u) \psi(v) \in E(H)$. La función $\psi$ es un isomorfismo de $G$ en $H$.

Un grafo $H$ es un subgrafo de $G$ si $V(H) \subseteq V(G)$ y $E(H) \subseteq E(G)$. Si $G$ es un grafo y $\emptyset \neq X \subseteq V(G)$, entonces el subgrafo inducido de $G$ por $X$ es un subgrafo $H$, tal que $V(H)=X$ y $E(H)$ es el conjunto de aristas de $G$ que tienen ambos extremos en $X$. El subgrafo inducido por $X$ es denotado por $G[X]$. Decimos que $G$ tiene al grafo $G[X]$ como subgrafo inducido. $H$ es un subgrafo inducido prohibido o simplemente subgrafo prohibido para una clase de grafos $\mathcal{C}$ cuando todo grafo $G$, que tiene al grafo $H$ como subgrafo inducido no pertenece a la clase $\mathcal{C}$. Decimos que $H$ es un subgrafo prohibido minimal para una clase de grafos $\mathcal{C}$ si $H$ es un sugrafo prohibido para $\mathcal{C}$ y $H-v \in \mathcal{C}$ para todo $v \in V(H)$.

Decimos que $G$ es un grafo sin el grafo $H$ cuando $G$ no tiene a $H$ como subgrafo inducido.

Un camino $P=\left\{v_{0}, v_{1}, . ., v_{k}\right\}$ es una secuencia no vacía de vértices distintos de $G$ tal que, para todo $i, 1 \leq i \leq k$, existe una arista $v_{i-1} v_{i}$ en $G$. Decimos entonces que $P$ es un camino entre $v_{0}$ y $v_{k}$ y que $P$ pasa por 
los vértices y por las aristas que tienen ambos extremos consecutivos en $P$. El entero $k$ determina la longitud de $P$. Cuando fuera más conveniente, un camino $P$ puede ser expresado por la secuencia $\left\{e_{1}, e_{2}, . ., e_{k}\right\}$ de las aristas por donde pasa. En cualquier caso, $v_{0}$ y $v_{k}$ son los extremos de $P$.

Dos caminos $P_{1}$ y $P_{2}$ comparten una arista $e$ si $P_{1}$ y $P_{2}$ pasan por $e$. En este caso también decimos que $P_{1}$ y $P_{2}$ tienen intersección en aristas.

Un ciclo $C=\left\{v_{0}, v_{1}, . ., v_{k}\right\}$ es una secuencia no vacía de vértices donde $\left\{v_{0}, v_{1}, . ., v_{k}\right\}$ es un camino, $v_{k-1} v_{k} \in E(G)$ y $v_{0}=v_{k}$. Un ciclo es par o impar cuando su longitud $k$ es par o impar, respectivamente. La distancia $d(u, v)$ entre dos vértices $u$ y $v$ de $G$ es la longitud del menor camino entre $u$ y $v$ en $G$.

Un grafo $G$ es conexo si entre cualquier par de vértices distintos de $G$ existe un camino. Una componente conexa de $G$ es un subgrafo conexo maximal de $G$. Un grafo $G$ es disconexo cuando no es conexo. Los grafos usados en este trabajo serán conexos, a menos que se diga lo contrario.

Un árbol es un grafo conexo que no posee ciclos. Un subgrafo conexo de un árbol es llamado un subárbol. Un vértice de grado uno en un árbol es llamado una hoja. Decimos que el árbol es de grado $h$ cuando el grado máximo de sus vértices es igual a $h$. Una arista de un árbol $T$ es una arista pendiente si uno de sus extremos es una hoja de $T$.

Un grafo $G$ es completo si cualesquiera dos vértices distintos de $G$ son adyacentes. Excepto por isomorfismos, existe un único grafo completo con $n$ vértices y tal grafo es denotado por $K_{n}$.

Si $G$ es un grafo, el complemento de $G$, denotado por $\bar{G}$, es un grafo cuyo conjunto de vértices es $V(G)$ y tal que dados dos vértices distintos $u$ y 
$v$, se tiene que $u v \in E(\bar{G})$ si y solamente si $u v \notin E(G)$.

Un conjunto completo es un subconjunto de vértices mutuamente adyacentes. Un clique es un conjunto completo maximal. La familia de cliques de $G$ es denotada por $\mathcal{C}(G)$. Un conjunto estable es un subconjunto de vértices no adyacentes de a pares.

Sea $C=\left\{v_{0}, v_{1}, . ., v_{k}\right\}$ un ciclo en $G$ y $e=u v \in E(G)$, entonces $e$ es una cuerda en $C$ si $u$ y $v$ son vértices de $C, e \neq v_{i-1} v_{i}$ para todo $i, 1 \leq i \leq k$. Un ciclo de longitud $k$ sin cuerdas es denotado por $C_{k}$. Un grafo $G$ es cordal si todo ciclo $C$ en $G$, con por lo menos cuatro vértices, tiene una cuerda.

Una coloración de $V(G)$ es una coloración de los vértices de $G$ y tal que vértices adyacentes reciben distinto color. El número cromático de un grafo $G$, denotado por $\chi(G)$, es el menor número de colores en una colarción de $G$.

Un grafo $G$ es perfecto cuando el número cromático de cada subgrafo inducido es igual al tamaño del mayor clique de ese subgrafo. Se sabe que todo grafo cordal es perfecto [17] .

Sea $P$ una familia de caminos en un árbol huésped $T$. Se definen dos tipos de grafos de intersección con el par $\langle P, T\rangle$, llamados grafos VPT y grafos EPT.

El grafo arista-intersección de $\mathbf{P}, E P T(P)$, tiene vértices que corresponden a los miembros de $P$, y dos vértices son adyacentes en $E P T(P)$ si y sólo si los correspondientes caminos en $P$ comparten al menos una arista (dos vértices adyacentes) en $T$. Un grafo no dirigido $G$ es llamado un grafo arista-intersección de caminos en un árbol (EPT) si $G=$ 
EPT $(P)$ para algún $P$ y $T$, y $\langle P, T\rangle$ es llamada una representación EPT de $G$. Cuando el grado máximo del árbol huésped es $h$ la representación EPT de $G$ es llamada una $(\mathbf{h}, \mathbf{2 , 2})$-representación de $G$. La clase de grafos que admite una $(h, 2,2)$-representación se denota $[\mathbf{h , 2 , 2 ]}$. Notar que la clase de los grafos EPT es la unión de las clases $[h, 2,2]$ para $h \geq 2$. De ahí que, $\mathrm{EPT}=[\infty, 2,2]$. Los grafos EPT son usados en aplicaciones de redes, donde el problema de planificación de llamadas no dirigidas en una red que es un árbol es equivalente al problema de colorear un grafo EPT. La red de comunicación está representada como un grafo no dirigido de interconexión, donde cada arista es asociada con una conexión física entre nodos. Una llamada no dirigida es un camino en la red. Cuando la red es un árbol, este modelo es claramente una representación EPT. Colorear el grafo EPT de forma tal que dos vértices adyacentes tengan distintos colores, significa que llamadas no dirigidas que comparten una conexión física tienen que planificarse en distintos momentos.

Similarmente, el grafo vértice-intersección de $\mathbf{P}, V P T(P)$, tiene vértices que corresponden a los miembros de $P$, y dos vértices son adyacentes en $V P T(P)$ si y sólo si los correspondientes caminos en $P$ comparten al menos un vértice en $T$. Un grafo no dirigido $G$ es llamado un grafo vérticeintersección de caminos en un árbol (VPT) si $G=V P T(P)$ para algún $P$ y $T$, y $\langle P, T\rangle$ es llamada una representación VPT de $G$. Cuando el grado máximo del árbol huésped $T$ es $h$, la representación VPT de $G$ es llamada una (h, 2,1)-representación de $G$. La clase de grafos que admite una $(h, 2,1)$ representación se denota $[\mathbf{h}, \mathbf{2 , 1}]$. Notar que la clase de los grafos VPT es la unión de las clases $[h, 2,1]$ para $h \geq 2$. De ahí que $\mathrm{VPT}=[\infty, 2,1]$. Los grafos 
VPT son útiles en muchas áreas, entre las cuales cabe destacar la genética, arqueología y ecología.

Decimos que una familia satisface la propiedad de Helly cuando para toda subfamilia cuyos miembros se intersectan dos a dos se tiene que la intersección de esta subfamilia es no vacía.

Dado que una familia de caminos en un árbol huésped $T$ satisface la propiedad de Helly [8], si $C$ es un clique de $G$ entonces existe un vértice $q$ de $T$ tal que $C=\left\{v \in V(G) \mid q \in V\left(P_{v}\right)\right\}$. Por otro lado, si $q$ es cualquier vértice del árbol huésped $T$, el conjunto $\left\{v \in V(G) \mid q \in V\left(P_{v}\right)\right\}$, denotado por $\mathbf{C}_{\mathbf{q}}$, es un conjunto completo de $G$, pero no necesariamente un clique. Para evitar este inconveniente, se introduce la noción de representación llena en $q$.

Sea $\langle\mathcal{P}, T\rangle$ una representación VPT de $G$ y sea $q$ un vértice de grado $h$ de $T$. Las componentes conexas de $T-q$ son llamadas las ramas de $T$ en $q$. Un camino está contenido en una rama si todos sus vértices son vértices de la rama. Notar que si $N_{T}(q)=\left\{q_{1}, q_{2}, . ., q_{h}\right\}$ entonces $T$ tiene exactamente $h$ ramas en $q$. La rama que contiene a $q_{i}$ se denota $T_{i}$; decimos que $q_{i}$ es la raíz de $T_{i}$. Las ramas $T_{i}$ y $T_{j}$ están unidas por un camino $P_{v} \in \mathcal{P}$ si ambos vértices $q_{i}$ y $q_{j}$ pertenecen a $V\left(P_{v}\right)$.

Definición 2.1 Una representación $V P T,\langle\mathcal{P}, T\rangle$, es llena en un vértice $\boldsymbol{q}$ de $\boldsymbol{T}$ si, para cualesquiera dos ramas $T_{i}$ y $T_{j}$ de $T$ en $q$, existen caminos $P_{v}, P_{w}, P_{u} \in \mathcal{P}$ tal que:

1. las ramas $T_{i}$ y $T_{j}$ están unidas por $P_{v}$;

2. $P_{w}$ está contenido en $T_{i}$ e intersecta a $P_{v}$ en al menos un vértice; $y$ 
3. $P_{u}$ está contenido en $T_{j}$ e intersecta a $P_{v}$ en al menos un vértice. Una representación $\langle\mathcal{P}, T\rangle$ es llena si es llena en todo $q \in V(T)$ con $d_{T}(q) \geq 4$.

Una consequencia clara de la definición previa es que si $\langle\mathcal{P}, T\rangle$ es llena en un vértice $q$ de $T$, con $d_{T}(q)=h \geq 3$, entonces $C_{q}$ es un clique de $G$. 


\section{Capítulo 3}

\section{Reconocimiento de los grafos vértice-interseción de caminos en árboles de grado acotado}

Los resultados presentados en este Capítulo fueron publicados en [3].

En este Capítulo, nos enfocamos en las clases $[h, 2,1]$ para cualquier $h \geq 3$ fijo; ellas son todas subclases de $V P T$. Caracterizamos a los grafos $[h, 2,1]$ usando el número cromático. Mostramos que el problema de decidir si un grafo $V P T$ dado pertenece a $[h, 2,1]$ es NP-completo, mientras que el problema de decidir si el grafo dado pertenece a $[h, 2,1]-[h-1,2,1]$ es NP-difícil. Ambos problemas permanecen NP-difíciles aún cuando nos restringimos a la clase VPT $\cap$ Split. Adicionalmente, presentamos una subclase no trivial de VPT $\cap$ Split en la cual éstos problemas son polinomiales. No consideramos el caso $h=2$ pues $[2,2,1]=$ Intervalos. Nuestros resultados se aplican para cualquier $h \geq 3$ y pueden ser vistos como una generalización del caso $h=3$ 
el cual conduce a la clase $[3,2,1]=[3,2,2]=$ EPT $\cap$ Cordal considerado en $[15,35]$.

\subsection{Resultados básicos}

El siguiente Teorema y su Corolario muestran que una representación VPT que no es llena se puede modificar hasta obtener una representación VPT llena sin aumentar el grado máximo del árbo huésped; y, más aún, disminuyendo el grado de los vértives de $T$ en los cuales la representación no es llena.

Teorema 3.1 Sea $G$ un grafo y $\langle\mathcal{P}, T\rangle$ una representación VPT de G. Supongamos que existe un vértice $q \in V(T)$ con $d_{T}(q)=h \geq 4$ y dos ramas de $T$ en $q$ las cuales no están unidas por un camino de $\mathcal{P}$. Entonces existe una representación $V P T,\left\langle\mathcal{P}^{\prime}, T^{\prime}\right\rangle$, de $G$ con $V\left(T^{\prime}\right)=V(T) \cup\left\{q^{\prime}\right\}, q^{\prime} \notin V(T), y$

$$
d_{T^{\prime}}(x)= \begin{cases}3 & \text { si } x=q^{\prime} \\ h-1 & \text { si } x=q \\ d_{T}(x) & \text { si } x \in V\left(T^{\prime}\right) \backslash\left\{q, q^{\prime}\right\}\end{cases}
$$

Demostración: Sean $q_{1}$ y $q_{2}$ los vecinos de $q$ que son raíces de dos ramas que no están unidas. Obtenemos la representación $\left\langle\mathcal{P}^{\prime}, T^{\prime}\right\rangle$ de $G$ como sigue (ver Figura 3.1): el conjunto de vértices de $T^{\prime}$ es $V(T) \cup\left\{q^{\prime}\right\}$, donde $q^{\prime}$ es un nuevo vértice. El conjunto de aristas es $\left(E(T) \backslash\left\{q q_{1}, q q_{2}\right\}\right) \cup\left\{q^{\prime} q_{1}, q^{\prime} q_{2}, q^{\prime} q\right\}$. Observar que el grado de cada vértice $x \in V\left(T^{\prime}\right)$ es el requerido en el presente teorema. Ahora definimos los caminos $P_{v}^{\prime}$ para $v \in V(G)$ : si $q_{1}$ y $q$ o $q_{2}$ y $q$ pertenecen a $V\left(P_{v}\right)$ entonces $V\left(P_{v}^{\prime}\right)=V\left(P_{v}\right) \cup\left\{q^{\prime}\right\}$. En cualquier 
otro caso, $V\left(P_{v}^{\prime}\right)=V\left(P_{v}\right)$. Dado que ningún camino de $\mathcal{P}$ contiene a ambos vértices $q_{1}$ y $q_{2}$, cada $V\left(P_{v}^{\prime}\right)$ induce efectivamente un camino en $T^{\prime}$. Más aún, dado que todos los caminos donde el vértice $q^{\prime}$ fue agregado tenían el vértice $q$ en común, es claro que, para cualquier par de vértices $v, w \in V(G)$, $V\left(P_{v}\right) \cap V\left(P_{w}\right) \neq \emptyset$ si y sólo si $V\left(P_{v}^{\prime}\right) \cap V\left(P_{w}^{\prime}\right) \neq \emptyset$. Se tiene que $\left\langle\mathcal{P}^{\prime}, T^{\prime}\right\rangle$ es una representación VPT de $G$ y la prueba está completa.
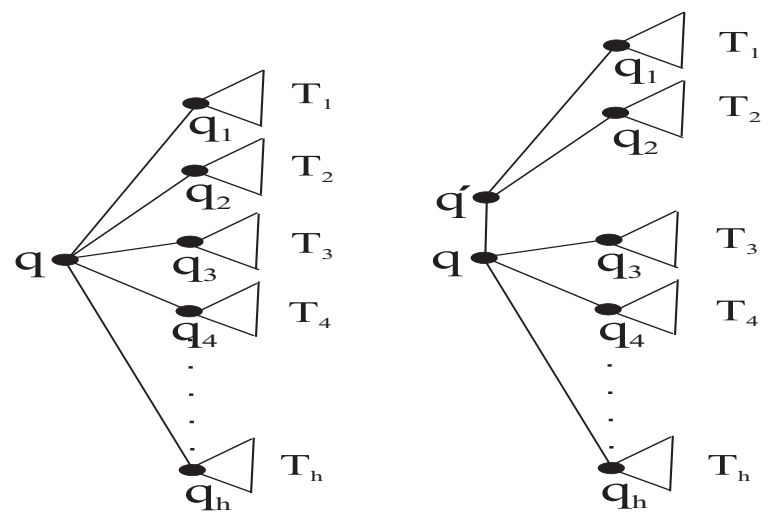

Figura 3.1: Construcción de $\left\langle\mathcal{P}^{\prime}, T^{\prime}\right\rangle$ a partir de $\langle\mathcal{P}, T\rangle$ en el Teorema 3.1.

Corolario 3.1 Cualquier grafo $[h, 2,1]$ admite una $(h, 2,1)$-representación llena.

Demostración: Sea $\langle\mathcal{P}, T\rangle$ una $(h, 2,1)$-representación de $G$. Podemos asumir, sin pérdida de generalidad, que si $x$ es un vértice extremo de un camino $P_{v} \in \mathcal{P}$ entonces existe un camino $P_{u} \in \mathcal{P}$ que intersecta a $P_{v}$ sólo en $x$, en otro caso el vértice $x$ puede ser removido de $P_{v}$. Esto implica que cualquier camino $P$ uniendo dos ramas intersecta a caminos contenidos en esas ramas. Luego, la prueba sale inductivamente aplicando el Teorema 3.1 en 
un vértice $q$ de $T$ de grado al menos cuatro en el cual $\langle\mathcal{P}, T\rangle$ no es llena. Más claramente, supongamos que $q \in V(T)$ con $d_{T}(q) \geq 4$ tal que $\langle\mathcal{P}, T\rangle$ no es llena en $q$. Esto es, existen ramas $T_{i}, T_{j}$ de $T$ en $q$ las cuales no están unidas por un camino de $\mathcal{P}$. Por el Teorema 3.1 podemos hallar una nueva representación $\left\langle\mathcal{P}^{\prime}, T^{\prime}\right\rangle$ de $G$ (sin aumentar el grado de los vértices del árbol) la cual es llena en $q$. Luego, hacemos este procedimiento en cada vértice de $T$ de grado mayor o igual a cuatro, en el cual la representación no es llena. De esta manera, obtenemos un $(h, 2,1)$-representación llena de $G$.

Un grafo $G$ es split si $V(G)$ se puede particionar en un conjunto estable $S$ y un clique $K$ [7]. El par $(S, K)$ es una partición split de $G$ y esta partición es única salvo por isomorfismos. Los vértices en $S$ son llamados vértices estables, y $K$ es llamado el clique central de $G$. Un vértice $s$ es un vértice estable dominado si $s \in S$ y existe $s^{\prime} \in S$ tal que $N(s) \subseteq N\left(s^{\prime}\right)$. Notar que si $G$ es split entonces $\mathcal{C}(G)=\{K\} \cup\{N[s] \mid s \in S\}$. Llamaremos Split a la clase de los grafos que son split.

Lema 3.1 Sea $G \in V P T \cap$ Split con partición split $(S, K)$ y sea $\langle\mathcal{P}, T\rangle$ una representación VPT llena de $G$. Si $q \in V(T)$ y $C_{q} \neq K$, entonces $d_{T}(q) \leq 3$.

Demostración: Asumamos, para una contradición, que existe $q \in V(T)$ tal que $C_{q} \neq K$ y $d_{T}(q) \geq 4$. Dado que $\langle\mathcal{P}, T\rangle$ es llena y $d_{T}(q) \geq 4, C_{q}$ es un clique de $G$, esto es, existe $s \in S$ tal que $C_{q}=N[s]$. Dado que en $C_{q}$ existen al menos tres vértices que no pertenecen $K$, entonces $s$ tiene al menos dos vecinos que no están en $K$. Esto contradice el hecho de que $G \in$ Split. 
Un grafo es k-coloreable si sus vértices pueden ser pintados con a lo sumo $k$ colores de manera que dos vértices que son adyacentes reciban distinto color. El número cromático de $G$, denotado por $\chi(G)$, es el menor $k$ tal que $G$ es $k$-coloreable. Un vértice $v \in V(G)$ o una arista $e \in E(G)$ es un elemento crítico de $G$ si $\chi(G-v)<\chi(G)$ o $\chi(G-e)<\chi(G)$. Un grafo $G$ con número cromático $h$ es h-vértice crítico (resp. h-crítico) si cada uno de sus vértices (resp. aristas) es un elemento crítico.

Teorema 3.2 [27] Para cualquier $k \geq 3$ fijo, el problema de decidir si un grafo $G$ dado es k-coloreable es NP-completo.

Un grafo es perfecto si y sólo si no contiene ciclos impares de longitud al menos cinco, o su complemento, como subgrafos inducidos [10].

Teorema 3.3 [23] Para cualquier $k \geq 3$ fijo, el problema de decidir si un grafo perfecto $G$ dado es $k$-coloreable es polinomial.

\subsection{Caracterización de $[\mathbf{h}, 2, \mathbf{1}]$, para $h \geq 3$}

En esta Sección, presentamos una caracterización de los grafos VPT que pueden ser representados en un árbol huésped con grado máximo a lo sumo $h$. La caracterización es dada en términos del número cromático de los grafos ramas.

Definición 3.1 [15] Sea $C \in \mathcal{C}(G)$. El grafo rama de $G$ para el clique $C$, denotado por $B(G / C)$, es definido como sigue: sus vértices son los vértices de $V(G)-C$ que son adyacentes a al menos vértice de $C$, y dos vértices u y $v$ son adyacentes en $B(G / C)$ si y sólo si 
1. $u v \notin E(G)$;

2. existe un vértice $x \in C$ tal que $x u \in E(G)$ y $x v \in E(G)$;

3. existe un vértice $y \in C$ tal que yu $\in E(G)$ y yv $\notin E(G) ; y$

4. existe un vértice $z \in C$ tal que $z u \notin E(G)$ y $z v \in E(G)$.

Para la conveniencia del lector ofrecemos en la Figura 3.2 un ejemplo donde mostarmos que $B\left(A_{4} / K\right)=K_{4}$, y son destacados para las arista 12 de $K_{4}$ los correspondientes vértices $x, y, z$ de $A_{4}$.
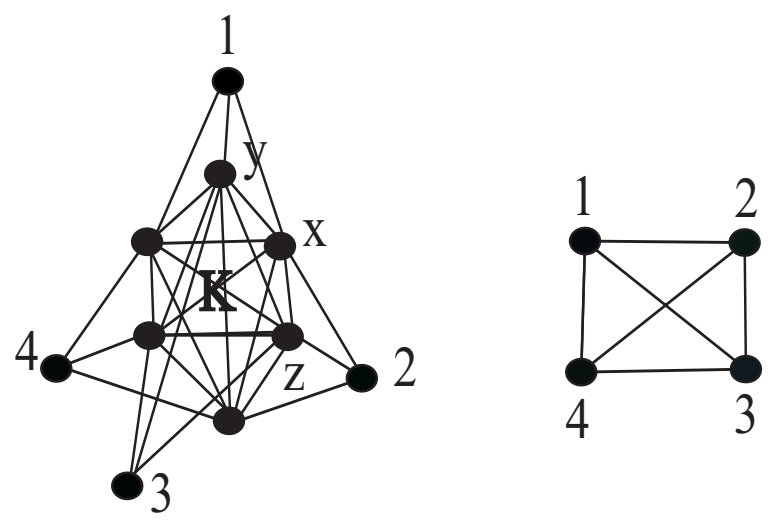

Figura 3.2: El grafo $A_{4}$ y el grafo rama $B\left(A_{4} / K\right)$.

Es claro que si $C \in \mathcal{C}(G)$ y $v \in V(G)-C$ entonces $C \in \mathcal{C}(G-v)$. El siguiente lema dice qué pasa con el grafo rama cuando removemos tales vértices. Su prueba es trivial.

Lema 3.2 Sea $C \in \mathcal{C}(G)$ y $v \in V(G)-C$ :

1. Si $v \notin V(B(G / C))$ entonces $B((G-v) / C)=B(G / C)$;

2. si $v \in V(B(G / C))$ entonces $B((G-v) / C)=B(G / C)-v$. 
Como será visto en lo que sigue, los grafos ramas de grafos VPT pueden ser usados para describir propiedades de las representaciones VPT.

Lema 3.3 Sea $G$ un grafo VPT. Sea $C$ un clique de $G,\left\langle\left(P_{v}\right)_{v \in V(G)}, T\right\rangle$ una representación VPT de $G$ y q un vértice de $T$ tal que $C=C_{q}$. Si v es un vértice de $B(G / C)$ entonces $P_{v}$ está contenido en alguna rama de $T$ en $q$. Si dos vértices $u$ y $v$ son adyacentes en $B(G / C)$ entonces $P_{u}$ y $P_{v}$ no están contenidos en una misma rama de $T$ en $q$.

Demostración: Por la definición de grafo rama, si $u \in V(B(G / C))$ entonces $u \notin C$. Se tiene que $q \notin V\left(P_{u}\right)$, esto es, $P_{u}$ está contenido en alguna rama de $T$ en $q$.

Sea $v$ adyacente a $u$ en $B(G / C)$. Supongamos por el absurdo que $P_{u}$ y $P_{v}$ están contenidos en una misma rama de $T$ en $q$. Sean $x$ e $y$ los vértices de $P_{u}$ y $P_{v}$ respectivamente que están a menor distancia de $q$. Dado que existe un vértice de $C$ que es adyacenete a $u$ y $v$, existe un camino en $T$ que contiene a $q, x$ e $y$. De ahí que, sin pérdida de generalidad, podemos asumir que $x$ está entre $q$ e $y$ o que $x=y$. En ambos casos, $N(v) \cap C \subseteq N(u) \cap C$, lo cual contradice el hecho de que $u$ y $v$ son adyacentes en $B(G / C)$.

Lema 3.4 Sea $\langle\mathcal{P}, T\rangle$ una representación VPT de $G$. Sea $C \in \mathcal{C}(G)$ y $q \in$ $V(T)$ tal que $C=C_{q}$. Si $d_{T}(q)=h$, entonces $B(G / C)$ es $h$-coloreable.

Demostración: Sean $T_{1}, T_{2}, . ., T_{h}$ las ramas de $T$ en $q$. Por el Lema 3.3, si coloreamos cada vértice $v$ de $B(G / C)$ con el índice $i$ de la rama $T_{i}$ que contiene al camino $P_{v}$, entonces obtenemos una coloración propia de $B(G / C)$. 
Dado que hay $h$ ramas, $B(G / C)$ es $h$-coloreable.

Teorema 3.4 Sea $G \in V P T$ y $h \geq 3$. El grafo $G$ pertenece a $[h, 2,1]$ si $y$ sólo si $B(G / C)$ es h-coloreable para todo $C \in \mathcal{C}(G)$. La implicación directa es verdadera también para $h=2$.

Demostración: Sea $\langle\mathcal{P}, T\rangle$ una $(h, 2,1)$-representación de $G$, con $h \geq 2$. Si $C \in \mathcal{C}(G)$ entonces existe $q \in V(T)$ tal que $C=C_{q}$. Dado que $d_{T}(q)=l \leq h$, por el Lema $3.4, B(G / C)$ es $l$-coloreable. En consecuencia, $B(G / C)$ es $h$ coloreable.

La recíproca para $h=3$ fue probada por Golumbic y Jamison en [15]; entonces podemos asumir que $h \geq 4$. En forma similar a lo hecho en [15], probaremos que si $B(G / C)$ es $h$-coloreable para todo clique $C$ de $G$, entonces $G$ admite una $(h, 2,1)$-representación.

Sea $\langle\mathcal{P}, T\rangle$ una representación VPT llena de $G$. Esta existe por el Corolario 3.1.

Procedemos por inducción en el número $k$ de vértices de $T$ cuyo grado es mayor que $h$. Si $k=0$ no hay nada que hacer. Si $k>0$, existe un vértice $q$ de $T$ con grado $d>h$. Dado que la representación es llena, $C_{q}$ es un clique de $G$.

Digamos que $N_{T}(q)=\left\{q_{1}, q_{2}, \ldots, q_{d}\right\}$ y, para cada $i$, sea $T_{i}$ la rama de $T$ en $q$ que contiene a $q_{i}$. Podemos asumir que, para cada $T_{i}$, existe un vértice $v_{i} \in V(G)$ tal que el camino correspondiente $P_{v_{i}}$ está contenido en la rama $T_{i}$ y $q_{i} \in V\left(P_{v_{i}}\right)$. De otra manera, podemos contraer la arista $q q_{i}$ para obtener una nueva representación VPT de $G$ sin cambiar la intersección de los 
caminos, y repetir este procedimiento tantas veces como sea necesario hasta que lo asumido valga. Notar que durante esta operación algunos vértices de $T$ desaparecen, y que el grado de $q$ podría aumentar, pero el número de vértices cuyo grado es más grande que $h$ no aumenta.

Decimos que $v_{i}$ es el líder de $T_{i}$ para $1 \leq i \leq d$. Observar que cada líder $v_{i}$ es un vértice del grafo rama $B\left(G / C_{q}\right)$. Sea $\mu_{i}$ el color de $v_{i}$ en una $h$ coloración propia de $B\left(G / C_{q}\right)$. Coloreamos cada rama $T_{i}$ con el color $\mu_{i}$ de su líder $v_{i}$.

Podemos asumir que si dos ramas $T_{i}$ y $T_{j}$ están unidas entonces tienen diferentes colores. Pues, supongamos por el absurdo, que $T_{i}$ y $T_{j}$ están unidas por un camino $P_{v} \in \mathcal{P}$ y tienen el mismo color. Entonces, sus líderes $v_{i}$ y $v_{j}$ tienen el mismo color en $B\left(G / C_{q}\right)$, lo cual implica que

$$
v_{i} \text { y } v_{j} \text { no son adyacentes en } B\left(G / C_{q}\right) \text {. }
$$

Por la definición del grafo rama y por (3.1), dado que $v_{i}$ y $v_{j}$ no son adyacentes en $G, v \in C_{q}$ y $v$ es adyacente a $v_{i}$ y a $v_{j}$ en $G$, podemos asumir, sin pérdida de generalidad, que $N\left(v_{i}\right) \cap C_{q} \subseteq N\left(v_{j}\right) \cap C_{q}$. Esto significa que la rama $T_{i}$ está unida sólo a la rama $T_{j}$; luego podemos cambiar el color de $T_{i}$ a cualquiera de los $h-1$ colores restantes. Repitiendo este procedimietno tantas veces como sea necesario, obtenemos una $h$-coloración de las ramas de modo que cualesquiera dos ramas que estén unidas tienen diferentes colores.

Ahora, obtenemos una nueva representación $\operatorname{VPT},\left\langle P^{\prime}, T^{\prime}\right\rangle$, de $G$ como sigue. El árbol $T^{\prime}$ es obtenido de $T$ a través del siguiente procedimiento (en la Figura 3.3 ofrecemos un ejemplo):

para todo $i, 1 \leq i \leq d$, removemos la arista $q q_{i} ;$

para todo $i, 1 \leq i \leq d$, agregamos un vértice $q_{i}^{\prime}$ adyacente a $q_{i} ; \mathrm{y}$ 
para todo $j, 1 \leq j \leq h$, agregamos las aristas necesarias para obtener un camino inducido con vértice extremo $q$ conectando los vértices $q_{i}^{\prime}$ tal que la rama correspondiente $T_{i}$ tiene color $\mu_{j}$.

El resto del árbol $T$ permanece sin cambios. Notar que $d_{T^{\prime}}(q)=h$, $d_{T^{\prime}}\left(q_{i}^{\prime}\right) \leq 3$ para todo $i$, y los vértices restantes tienen el mismo grado como vértices de $T^{\prime}$ que como vértices de $T$.

Los únicos caminos de $\mathcal{P}$ que son modificados para obtener los caminos de $\mathcal{P}^{\prime}$ son aquellos que contienen a $q$. Si un camino $P_{v} \in \mathcal{P}$ tiene a $q$ como vértice extremo e intersecta una rama $T_{i}$, entonces obtenemos $P_{v}^{\prime}$ reemplazando en $P_{v}$ la arista $q q_{i}$ por el único subcamino de $T^{\prime}$ uniendo $q$ y $q_{i}$. Si $P_{v}$ tiene a $q$ como un vértice interno, e intersecta dos ramas $T_{i}$ y $T_{j}$, entonces, obtenemos $P_{v}^{\prime}$ reemplazando en $P_{v}$ las aristas $q q_{i}$ y $q q_{j}$ por el único subcamino de $T^{\prime}$ uniendo $q_{i}, q$ y $q_{j}$. Notar que tal subcamino existe pues las ramas que están unidas tienen distintos colores, esto es, $q_{i}$ y $q_{j}$ están en distintas ramas de $T^{\prime}$ en $q$.

Es fácil de ver que la construcción descripta anteriormente deja el grafo de intersección de caminos sin cambios, mientras que el número de vértices cuyo grado es mayor que $h$, decrece. Luego, por inducción, la implicación queda probada.

Observar que la recíproca del Teorema 3.4 es falsa para $h=2$; consideremos, por ejemplo, el grafo obtenido de $K_{1,3}$ subdividiendo cada arista con un nuevo vértice. Este grafo, que llamamos $T_{2}^{3}$, es un subgrafo inducido prohibido para la clase de los grafos de intervalos. Es fácil de ver que $T_{2}^{3} \in \mathrm{VPT}$ y que $B(G / C)$ es 2 -coloreable para todo clique $C$; sin embargo, $T_{2}^{3} \notin[2,2,1]$. 


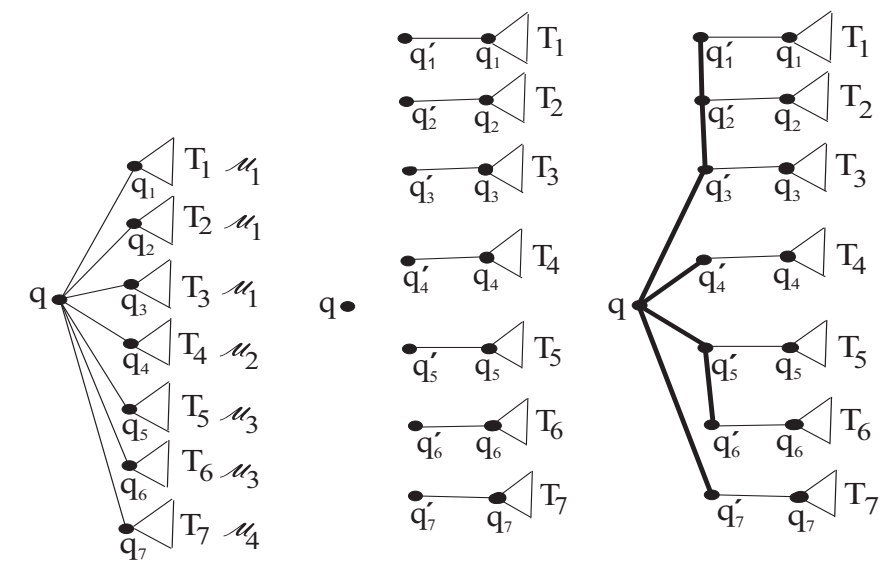

Figura 3.3: $B\left(G / C_{q}\right)$ es 4-coloreable con lo cual $G$ admite una $(4,2,2)$ representación.

Teorema 3.5 Sea $G \in V P T$ y $h \geq 4$. El grafo $G$ pertenece a $[h, 2,1]-[h-$ $1,2,1]$ si y sólo si $\operatorname{Max}_{C \in \mathcal{C}(G)}(\chi(B(G / C)))=h$. La recíproca es verdadera también para $h=3$.

Demostración: Por el Teorema 3.4, $G \in[h, 2,1]$ si y sólo si

$$
\operatorname{Max}_{C \in \mathcal{C}(G)}(\chi(B(G / C))) \leq h
$$

Y, por el mismo Teorema $3.4, G \notin[h-1,2,1]$ si y sólo si

$$
\operatorname{Max}_{C \in \mathcal{C}(G)}(\chi(B(G / C)))>h-1 .
$$




\subsection{Complejidad}

En esta Sección, probamos que, para cualquier $h \geq 3$, el problema de decidir si un grafo dado pertenece a $[h, 2,1]$ es NP-completo. También mostramos que reconocer a los grafos que están en $[h, 2,1]-[h-1,2,1]$ es NP-difícil para cualquier $h \geq 4$. Nuestros resultados prueban que ambos problemas permanecen difíciles aún cuando los grafos de entrada se restringen a VPT, split y sin vértices estables dominados.

Primero establecemos la siguiente propiedad fundamental de los grafos VPT $\cap$ Split la cual es usada en la prueba de los Teoremas 3.8 y 3.9.

Lema 3.5 Sea $G \in V P T \cap$ Split con partición split $(S, K)$, y sea $s \in S$. Entonces, $\chi(B(G / N[s])) \leq 3$.

Demostración: Sea $\langle P, T\rangle$ una representación VPT llena de $G$. Existe $q \in$ $V(T)$ tal que $C_{q}=N[s]$. Dado que $N[s] \neq K$, por el Lema 3.1, $d_{T}(q) \leq 3$. Luego, por el Lema 3.4, $\chi(B(G / N[s])) \leq d_{T}(q) \leq 3$.

Para la prueba de la NP-completitud, usamos una reducción al bien conocido problema de decidir si un grafo es $k$-coloreable citado en el Teorema 3.2 .

Dado un grafo $H$ construiremos en tiempo polinomial un grafo $G_{H} \in$ VPT $\cap$ Split sin vértices estables dominados de manera que $\chi(H)=h$ si y sólo si $G_{H} \in[h, 2,1]-[h-1,2,1]$.

Si $V(H)=\left\{v_{1}, v_{2}, \ldots, v_{n}\right\}$, entonces $G_{H}$ tiene el siguiente conjunto de vértices: 


$$
V\left(G_{H}\right)=\left\{\begin{array}{cl}
v_{i} & \text { para cada } 1 \leq i \leq n \\
v_{i j} & \text { para cada } 1 \leq i<j \leq n \text { tal que } v_{i} v_{j} \in E(H) \\
\tilde{v}_{i} & \text { para cada } 1 \leq i \leq n \operatorname{con} d_{H}\left(v_{i}\right)=1
\end{array}\right.
$$

Los cliques de $G_{H}$ son $K_{H}$ y $C_{v_{i}}$ para $1 \leq i \leq n$, donde:

$$
\begin{gathered}
K_{H}=\left\{v_{i j} \mid 1 \leq i<j \leq n\right\} \cup\left\{\tilde{v}_{i} \mid 1 \leq i \leq n y d_{H}\left(v_{i}\right)=1\right\} \\
C_{v_{i}}=\left\{v_{i}\right\} \cup\left\{v_{i j} \mid v_{j} \in N_{H}\left(v_{i}\right)\right\} \cup\left\{\tilde{v}_{i} \mid d_{H}\left(v_{i}\right)=1\right\} .
\end{gathered}
$$

Decimos que $G_{H}$ es el grafo construído a partir de $H$. Ver un ejemplo en la Figura 3.4.

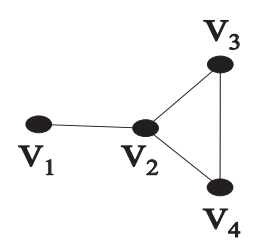

H

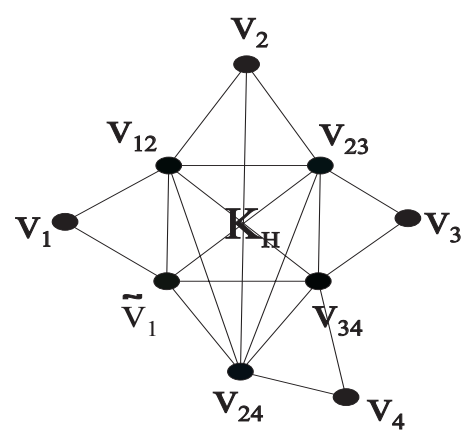

$\mathrm{G}_{\mathrm{H}}$

Figura 3.4: Un grafo $H$ y su correspondiente grafo $G_{H}$.

Notar que $G_{H}$ es un grafo split con el conjunto de vértices particionado en un conjunto estable $S_{H}$ de tamaño $n=|V(H)|$ correspondiendo a los 
vértices $v_{i}$, y un clique central $K_{H}$ de tamaño $|E(H)|+\mid\left\{v \in V(H) \mid d_{H}(v)=\right.$ $1\} \mid$ correspondiendo a los vértices restantes. La utilidad de $G_{H}$ se ve en las propiedades descriptas en los siguientes dos teoremas.

Teorema 3.6 1. $G_{H}$ es un grafo VPT $\cap$ Split sin vértices estables dominados; $2 . B\left(G_{H} / K_{H}\right)=H$.

Demostración: 1 . Por cómo fue construído $G_{H}$ es claro que es un grafo split sin vértices estables dominados.

2. Notar que $B\left(G_{H} / K_{H}\right)$ tiene exactamente $n$ vértices, que son justamente los vértices del grafo $H$. Esto es, $V\left(B\left(G_{H} / K_{H}\right)\right)=\left\{v_{1}, v_{2}, \ldots, v_{n}\right\}$.

Veremos que las aristas de $B\left(G_{H} / K_{H}\right)$ son exactamente las aristas de $H$. Supongamos que $v_{i} v_{j} \in E\left(B\left(G_{H} / K_{H}\right)\right)$ entonces, por la definición de grafo rama, existe un vértice de $K_{H}$ que es adyacente a ambos $v_{i}$ y $v_{j}$, llamaremos a este vértice $v_{i j}$. Ahora, por cómo fue construído el grafo $G_{H}$ se tiene que $v_{i} v_{j} \in E(H)$.

Recíprocamente, asumamos que $v_{i} v_{j} \in E(H)$, entonces $v_{i j}$ es un vértice de $K_{H}$ y es adyacente a $v_{i}$ y a $v_{j}$ en $G_{H}$. Afirmamos que existe un vértice de $K_{H}$ que es adyacente a $v_{i}$ pero no a $v_{j}$. Pues, si $d_{H}\left(v_{i}\right)=1$ entonces el vértice requerido de $K_{H}$ es $v_{i q}$. Si $d_{H}\left(v_{i}\right)>1$, $v_{i}$ debe tener un vecino $v_{l}$ con $l \neq j$, entonces el vértice requerido de $K_{H}$ es $v_{i l}$. En forma análoga, podemos probar que existe un vértice de $K_{H}$ adyacente a $v_{j}$ pero no a $v_{i}$. Dado que $v_{i} \mathrm{y}$ $v_{j}$ no son adyacentes en $G_{H}$, por la definición de grafo rama, obtenemos que $v_{i}$ y $v_{j}$ son adyacentes en $B\left(G_{H} / K_{H}\right)$. Con lo cual, $E\left(B\left(G_{H} / K_{H}\right)\right)=E(H)$ Concluímos que $B\left(G_{H} / K_{H}\right)=H$. 
Teorema 3.7 Sea $G_{H}$ el grafo obtenido de $H$ como antes. Para cualquier $h \geq 4, G_{H}$ pertenece $a[h, 2,1]-[h-1,2,1]$ si y sólo si $\chi(H)=h$.

Demostración: Dado que $h \geq 4$, por el Lema 3.5, $\operatorname{Max}_{C \in \mathcal{C}\left(G_{H}\right)} \chi\left(B\left(G_{H} / C\right)\right)=$ $\chi\left(B\left(G_{H} / K_{H}\right)\right)$, y, por el el Teorema 3.6, $\chi\left(B\left(G_{H} / K_{H}\right)\right)=\chi(H)$. En consecuencia, por el Teorema $3.5, G_{H}$ pertenece a $[h, 2,1]-[h-1,2,1]$ si y sólo si $\chi(H)=h$.

Teorema 3.8 Para cualquier $h \geq 4$, el problema de decidir si un grafo dado pertenece a $[h, 2,1]-[h-1,2,1]$ es NP-difícil, aún cuando nos restringimos a la clase de los grafos VPT $\cap$ Split sin vértices estables dominados.

Además, dado que una $(h, 2,1)$-representación es un certificado polinomial de pertenecer a $[h, 2,1]$; usando el Teorema 3.4 y la construcción anterior, hemos probado el siguiente resultado.

Teorema 3.9 Para cualquier $h \geq 3$, el problema de decidir si un grafo dado pertenece a $[h, 2,1]$ es NP-completo, aún cuando nos restringimos a la clase de los grafos VPT $\cap$ Split sin vértices estables dominados.

\subsubsection{Una subclase que es reconocible en tiempo po- linomial}

Hemos probado que decidir si dado un grafo VPT $\cap$ Split sin vértices estables dominados admite una representación como intersección de caminos de un árbol con grado máximo $h$ es un problema NP-completo. En lo que 
sigue describimos una subclase no trivial de VPT $\cap$ Split donde este problema se resuelve en tiempo polinomial.

Para $n \geq 4$, un $\mathbf{n}$-sol, denotado por $S_{n}$, es un grafo split con conjunto estable $\left\{s_{1}, s_{2}, . ., s_{n}\right\}$, clique central $\left\{v_{1}, v_{2}, . ., v_{n}\right\}$ tal que $N\left(s_{i}\right)=\left\{v_{i}, v_{i+1}\right\}$ para $1 \leq i \leq n-1$, y $N\left(s_{n}\right)=\left\{v_{n}, v_{1}\right\}$. En la Figura 3.5 mostramos algunos ejemplos.

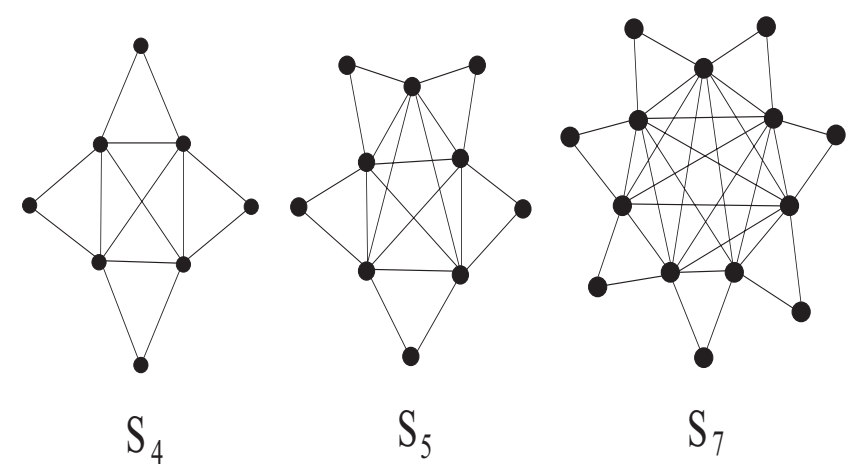

Figura 3.5: Los grafos soles $S_{4}, S_{5}$ y $S_{7}$.

Decimos que $G$ pertenece a SVS (special VPT subclass) si:

- $G \in \mathrm{VPT} \cap$ Split,

- para todo $v \in K,|N(v) \cap S| \leq 2$, donde $(S, K)$ es la partición split de $G$,

- si $S_{k}$, con $k \in\{4,2 n+1$ con $n \geq 2\}$, es inducido de $G$ entonces existe $v \in K$ tal que $v$ es adyacente a dos vértices no consecutivos del conjunto estable de $S_{k}$.

La clase SVS no es trivial, en el sentido de que incluye grafos en $[h, 2,1]$ para todo $h \geq 4$. 
Por ejemplo, para $n \geq 4$, sea $A_{n}$ (ver [19]) el grafo split con conjunto estable $S=\left\{s_{1}, . ., s_{n}\right\}$, clique central $K=\left\{v_{i j} \mid 1 \leq i<j \leq n\right\}$ y $N\left(v_{i j}\right)=$ $\left\{s_{i}, s_{j}\right\}$, para todo $1 \leq i<j \leq n$. Es fácil ver que $A_{n}$ pertenece a SVS, y $B\left(A_{n} / K\right)$ es un grafo completo con conjunto de vértices $\left\{s_{1}, . ., s_{n}\right\}$. En consecuencia, dado que $\operatorname{Max}_{C \in \mathcal{C}\left(A_{n}\right)} \chi\left(B\left(A_{n} / C\right)\right)=\chi\left(B\left(A_{n} / K\right)\right)=n$, por el Teorema 3.5, tenemos que $A_{n} \in[n, 2,1]-[n-1,2,1]$. (Como un ejemplo ver la Figura 3.6).

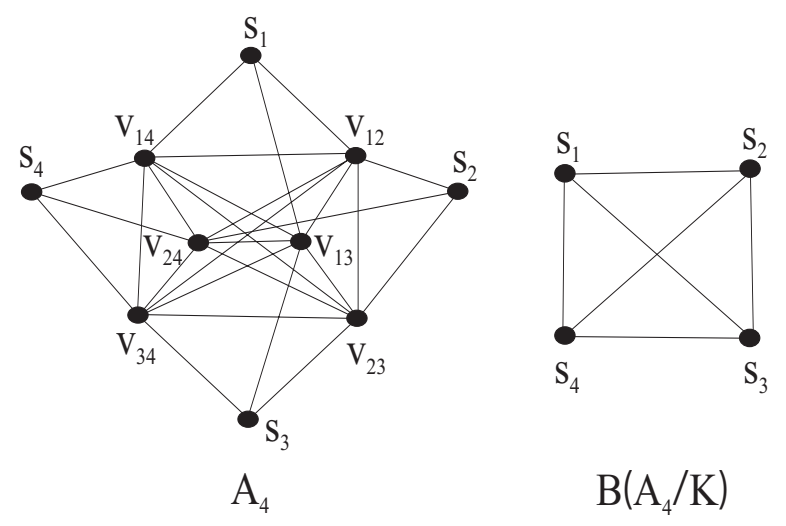

Figura 3.6: El grafo $A_{4}$ pertenece a SVS y $A_{4} \in[4,2,1]-[3,2,1]$.

Los siguientes dos lemas son usados en la prueba del Teorema principal 3.10 el cual establece que en la clase SVS, los grafos pertenecientes a $[h, 2,1]$ se pueden reconocer eficientemente, para cualquier $h \geq 4$

Lema 3.6 Sea $G \in V P T \cap$ Split con partición split $(S, K)$ tal que para todo $v \in K,|N(v) \cap S| \leq 2$, y sea $n \geq 4$. Si $B(G / K)$ tiene un $C_{n}$ como inducido entonces $G$ tiene un $S_{n}$ como inducido.

Demostración: Sea $\langle\mathcal{P}, T\rangle$ una representación VPT de $G$ y sea $q \in V(T)$ tal que $K=C_{q}$. Sea $C_{n}$ un ciclo inducido de $B(G / K)$ con vértices $s_{1}, s_{2}, \ldots, s_{n}$. 
Es claro que todo $s_{i} \in S$. Como $s_{i}$ es adyacente a $s_{i+1}$ en $B(G / K)$, existe $v_{i} \in K$ tal que $v_{i}$ es adyacente a $s_{i}$ y a $s_{i+1}$ en $G$. Además, dado que, para todo $v \in K,|N(v) \cap S| \leq 2$, se tiene que si $i, i^{\prime} \in\{1,2, . ., n\}$ y $i \neq i^{\prime}$ entonces $v_{i} \neq v_{i^{\prime}}$, con lo cual $s_{1}, s_{2}, \ldots, s_{n}, v_{1}, v_{2}, \ldots, v_{n}$ inducen un $n$-sol en $G$ y la prueba queda completa.

Lema 3.7 Si $G \in S V S$ con partición split $(S, K)$, entonces $B(G / K)$ es perfecto.

Demostración: Supongamos por el absurdo que $B(G / K)$ no es perfecto, entonces $B(G / K)$ tiene un ciclo impar o su complemento como subgrafos inducidos. Dado que el complemento de $C_{5}$ es $C_{5}$; y el complemento de cualquier ciclo impar de tamañoo 7 o más tiene como inducido un $C_{4}$, se tiene que $B(G / K)$ tiene como inducido un $C_{k}$, para algún $k \in\{4,2 n+1$ con $n \geq 2\}$. De ahí que, por el Lema 3.6, $G$ tiene un $S_{k}$ como inducido. Dado que $G \in$ SVS, existe $v \in K$ tal que $v$ es adyacente a dos vértices no consecutivos $s$ y $s^{\prime}$ del conjunto estable de $S_{k}$. Notar que la existencia de $v$ implica que los vértices $s$ y $s^{\prime}$ son adyacentes en $B(G / K)$. Esto contradice el hecho de que $C_{k}$ es un ciclo inducido de $B(G / K)$.

Teorema 3.10 Para cualquier $h \geq 4$ fijo, el problema de decidir si un grafo dado $G \in$ SVS pertenece a $[h, 2,1]-[h-1,2,1]$ es polinomial.

Demostración: Dado $G \in \mathrm{SVS}$, para determinar si $G \in[h, 2,1]-[h-1,2,1]$, por el Teorema 3.5 y el Lema 3.5, es suficiente con calcular el número cromático de $B(G / K)$, donde $K$ es el clique central de $G$. Notar que el grafo rama 
$B(G / K)$ puede ser obtenido en tiempo polinomial. Por otro lado, por el Lema 3.7, $B(G / K)$ es perfecto. Luego, por el Teorema 3.3, su número cromático puede ser calculado en tiempo polinomial. 


\section{Capítulo 4}

\section{Caracterización de los grafos $[\mathrm{h}, 2,1]$ por subgrafos inducidos prohibidos minimales}

Un método de caracterización de clases de grafos que es ampliamente empleado es el que da la definición por prohibición de subgrafos inducidos. Este método consiste en caracterizar una clase de grafos especificando el conjunto más simple de grafos que no pertenecen a la clase que se quiere caracterizar, y tal que los elementos de la clase son exactamente aquellos que no contienen ningún grafo del conjunto especificado como subgrafo inducido.

Las definiciones por prohibición son aplicables en diversas áreas de la Matemática [22]. Estas son interesantes tanto desde el punto de vista teórico como desde el punto de vista práctico, pues condensan mucha información sobre la estructura de la clase en un enunciado conciso, y esta información, muchas veces, da origen a algoritmos efecientes para la solución de diversos 
problemas que son difíciles en general [7].

Una caracterización de la clase VPT por prohibición fue obtenida parcialmente por M. Gutierrez, S. B. Tondato y J. L. Szwarcfiter en 2005 [24] y dada de manera completa por B. Lévêque, F. Maffray y M. Preissman en 2009 [29]. La prueba de dicha caracterización se basa en el Teorema del Arbol Característico [31], y en propiedades especiales de esos árboles para grafos VPT. Sin embargo, como la prueba es muy específica no entraremos en mayores detalles en cuanto a ella.

Teorema 4.1 (Caracterización de VPT por prohibidos) Un grafo es VPT si y sólo si no contiene a ninguno de los miembros de las familias $F_{0}, . ., F_{16}$ como subgrafos inducidos. (Ver Figura 4.1).

En este Capítulo caracterizamos la clase $[h, 2,1]$ por subgrafos inducidos prohibidos minimales para cada $h \geq 3$ fijo.

Parte de los resultados que aquí presentamos fueron publicados en [4]. El resto, está siendo evaluado para su futura publicación (ver [5]).

\subsection{Preliminaries}

Definición 4.1 Un clique $K$ de un grafo $G$ es llamado principal si

$$
\operatorname{Max}_{C \in \mathcal{C}(G)}(\chi(B(G / C)))=\chi(B(G / K)) .
$$

Lema 4.1 Si $(S, K)$ es la partición split de $G \in V P T \cap$ Split, entonces $K$ es un clique principal de $G$. 


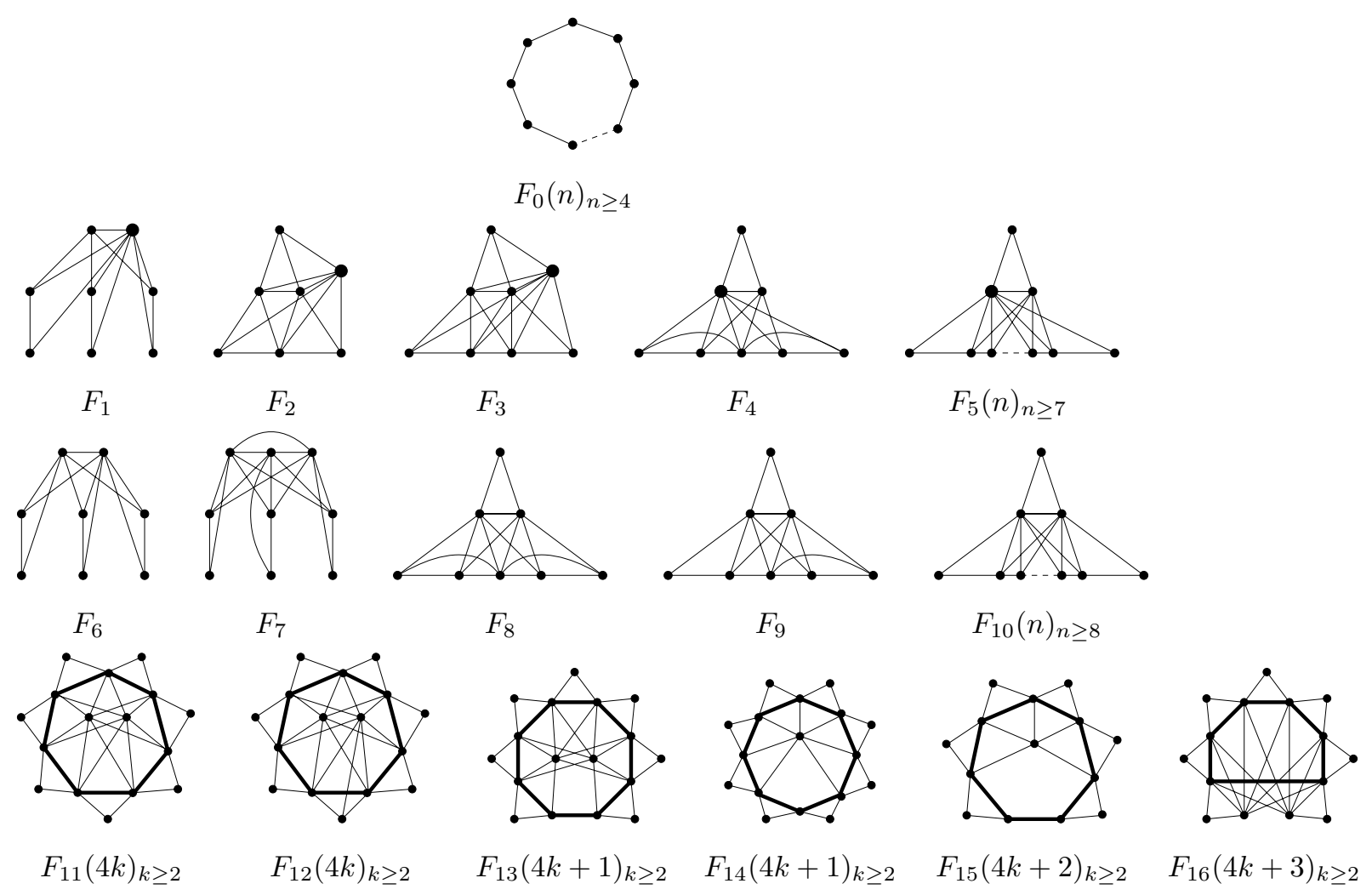

Figura 4.1: Subgrafos inducidos prohibidos minimales para VPT (los vértices en el ciclo marcados con aristas negritas forman un clique).

Demostración: Sea $s \in S$, sabemos que $N[s] \in \mathcal{C}(G)$. Observar que $V(B(G / N[s]))$ $=(K-N(s)) \cup S^{\prime}$, con $S^{\prime}=\{x \in S \mid N(x) \cap N(s) \neq \emptyset\}$. Afirmamos que los vértices de $K-N(s)$ son aislados en $B(G / N[s])$. Pues, sea $x \in K-N(s)$; si $y \in K-N(s)$, entonces $x y \notin E(B(G / N[s]))$ ya que $x y \in E(G)$ y, si $y \in S^{\prime}$ entonces $x y \notin E(B(G / N[s]))$ ya que $N(y) \subseteq N[x]$. Luego, el número cromático de $B(G / N[s])$ es igual al número cromático del subgrafo de $B(G / K)$ inducido por $S^{\prime}$, el cual es claramente un subgrafo de $B(G / K)$. Esto es, $\chi(B(G / N[s])) \leq \chi(B(G / K))$. En consecuencia, $\operatorname{Max}_{C \in \mathcal{C}(G)}(\chi(B(G / C)))=$ $\chi(B(G / K))$, con lo cual, $K$ es un clique principal de $G$. 


\subsection{Condiciones necesarias para grafos VPT que son minimales no- $[\mathrm{h}, 2,1]$}

En esta Sección damos condiciones necesarias para ser un grafo VPT minimal no- $[h, 2,1]$, con $h \geq 3$. El resultado más importante de esta Sección es el Teorema 4.4 el cual nos dirá que dado un grafo $G \in V P T$ y $h \geq 3$, si $G$ es un grafo minimal no- $[h, 2,1]$ entonces $G \in$ Split sin vértices estables dominados.

Recordemos que:

Definición 4.2 Un grafo minimal no-[h,2,1] es un subgrafo inducido prohibido minimal para la clase $[h, 2,1]$, esto significa un grafo $G$ tal que $G \notin[h, 2,1]$ pero $G-v \in[h, 2,1]$ para cualquier vértice $v \in V(G)$.

Teorema 4.2 Sea $G \in V P T$ y $h \geq 3$. Si $G$ es un grafo minimal no- $[h, 2,1]$ entonces $G \in[h+1,2,1]$.

Demostración: Sea $C \in \mathcal{C}(G)$ y $v \notin C$. Sabemos que $G-v \in[h, 2,1]$ entonces, por el Teorema 3.5, $\chi(B((G-v) / C)) \leq h$. Por el Lema 3.2, $\chi(B((G-$ $v) / C)) \geq \chi(B(G / C)-v) \geq \chi(B(G / C))-1$. Esto es, $\chi(B(G / C))-1 \leq h$ y entonces $\chi(B(G / C)) \leq h+1$. Luego, por el Teorema 3.4, $G \in[h+1,2,1]$. $\square$

Teorema 4.3 Sea K un clique principal de un grafo VPT minimal no $[h, 2,1]$ $G$, con $h \geq 3$. Luego: 
1. $V(B(G / K))=V(G)-K$

2. si $v \in V(G)-K$ entonces $|N(v) \cap K|>1$;

3. $B(G / K)$ es $(h+1)$-vértice crítico;

4. si $s_{1}, s_{2} \in V(G)-K$ entonces $N\left(s_{1}\right) \cap K \neq N\left(s_{2}\right) \cap K$.

5. $K-\{k\} \in \mathcal{C}(G-k)$ para todo $k \in K$.

Demostración: Por el Teorema 4.2, $G \in[h+1,2,1]$. Como $G \notin[h, 2,1]$, por el Teorema 3.5, dado que $K$ es un clique principal de $G$, tenemos que

$$
\chi(B(G / K))=h+1 .
$$

1. Es claro que $V(B(G / K)) \subseteq V(G)-K$. Supongamos que existe $v \in$ $V(G)-K$ tal que $v \notin V(B(G / K))$. Entonces, por el Lema 3.2, $B((G-$ $v) / K)=B(G / K)$. Dado que $G$ es un grafo minimal no-[h,2,1], $G-v \in$ $[h, 2,1]$ y, por el Teorema $3.4, B((G-v) / K)$ es $h$-coloreable. Esto es, $B(G / K)$ es $h$-coloreable lo cual contradice la condición (4.1).

2. Sea $v \in V(G)-K$. Por el item 1., sabemos que $v \in V(B(G / K))$; entonces $|N(v) \cap K| \geq 1$. Si $|N(v) \cap K|=1$, v sería un vértice aislado de $B(G / K)$ y $\chi(B(G / K))=\chi(B(G / K)-v)$. Pero, por el Lema 3.2 y el Teorema 3.4, dado que $G-v \in[h, 2,1]$ tenemos que $\chi(B(G / K)-v)=$ $\chi(B((G-v) / K)) \leq h$, lo cual también contradice la condición (4.1).

3. Por la condición (4.1), $\chi(B(G / K))=h+1$; asumamos, para obtener una contradicción, que $B(G / K)$ no es $(h+1)$-vértice crítico; entonces existe $v \in V(B(G / K))$ tal que $\chi(B(G / K)-v)=h+1$. Entonces, dado que $v \in$ $V(B(G / K))$, por el Lema 3.2, $\chi(B((G-v) / K))=\chi(B(G / K)-v)=h+1$, lo cual contradice que $G$ es un grafo minimal no- $[h, 2,1]$. 
4. Veremos que si $N\left(s_{1}\right) \cap K=N\left(s_{2}\right) \cap K$ entonces $s_{1} s_{2} \notin E(B(G / K))$ y $N_{B(G / K)}\left(s_{1}\right)=N_{B(G / K)}\left(s_{2}\right)$, lo cual contradice que $B(G / K)$ es $(h+1)$ vértice crítico. Pues, si $N\left(s_{1}\right) \cap K=N\left(s_{2}\right) \cap K$ entonces $s_{1} s_{2} \notin E(B(G / K))$ por la definición del grafo rama. Más aún, si $s_{3} \in N_{B(G / K)}\left(s_{1}\right)$ entonces existen $k_{1}, k_{2}, k_{3} \in K$ tales que: $k_{1} s_{1} \in E(G), k_{1} s_{3} \in E(G) ; k_{2} s_{1} \in E(G)$, $k_{2} s_{3} \notin E(G) ; k_{3} s_{1} \notin E(G), k_{3} s_{3} \in E(G)$. Y, dado que $N\left(s_{1}\right) \cap K=N\left(s_{2}\right) \cap K$, se tiene que $k_{1} s_{2} \in E(G), k_{2} s_{2} \in E(G), k_{3} s_{2} \notin E(G)$. Además, $s_{3} s_{2} \notin$ $E(G)$ porque en otro caso habría un 4-ciclo inducido $\left\{s_{2}, k_{2}, k_{3}, s_{3}\right\}$ en $G$, lo cual contradice que $G \in \mathrm{VPT}$ (ver Figura 4.1). En consecuencia, $s_{3} \in$ $N_{B(G / K)}\left(s_{2}\right)$; con lo cual hemos probado que $N_{B(G / K)}\left(s_{1}\right) \subseteq N_{B(G / K)}\left(s_{2}\right)$. De manera similar, es fácil ver que $N_{B(G / K)}\left(s_{2}\right) \subseteq N_{B(G / K)}\left(s_{1}\right)$.

5. Sea $\langle\mathcal{P}, T\rangle$ una $(h+1,2,1)$-representación de $G$ y sea $q \in V(T)$ tal que $K=C_{q}$. Afirmamos que $\langle\mathcal{P}, T\rangle$ es llena en $q$. Pues, supongamos, para una contradicción, que $\langle\mathcal{P}, T\rangle$ no es llena en $q$. Podemos asumir, sin pérdida de generalidad, que si $x$ es un extremo de un camino $P_{v} \in \mathcal{P}$ entonces existe un camino $P_{u} \in \mathcal{P}$ intersectando a $P_{v}$ sólo en $x$, en otro caso el vértice $x$ podría removerse de $P_{v}$. Esto implica que cualquier camino de $\mathcal{P}$ uniendo dos ramas intersecta caminos contenidos en esas ramas. Luego, dado que $\langle\mathcal{P}, T\rangle$ no es llena en $q$, existen ramas $T_{i}$ y $T_{j}$ de $T$ en $q$ las cuales no están unidas por un camino de $\mathcal{P}$. Entonces, por el Teorema 3.1, podemos obtener un nueva representación VPT, $\left\langle\mathcal{P}^{\prime}, T^{\prime}\right\rangle$, de $G$ con $d_{T^{\prime}}(q) \leq h$. Esto es, por el Lema 3.4, $B\left(G / C_{q}\right)$ es $h$-coloreable lo cual contradice que $C_{q}$ es un clique principal de $G$.

Luego, como $\langle\mathcal{P}, T\rangle$ es llena en $q$, cualquier par de ramas de $T$ en $q$ están unidas por un camino de $\mathcal{P}$. Si existiera $k \in C_{q}$ tal que $C_{q}-\{k\}$ no es un 
clique de $G-k$, existiría $v \in V(G)-C_{q}$ tal que $v$ es adyacente a todos los vértices de $C_{q}-\{k\}$. Sean $T_{1}, T_{2}, . ., T_{h+1}$ las ramas de $T$ en $q$. Asumamos, sin pérdida de generalidad, que $P_{k}$ está contenido en $\{q\} \cup T_{1} \cup T_{2}$. Como $v \in V(G)-C_{q}$, existe $i$ tal que $P_{v}$ está contenido en $T_{i}$. Y, como $h \geq 3$, existe una rama $T_{s}$, con $s \neq 1,2, i$. Sea $P_{u}$ el camino de $\mathcal{P}$ uniendo $T_{s}$ y $T_{r}$, con $r \neq i$. Es claro que $u \in C_{q}$ y $v$ no es adyacente a $u$, lo cual contradice que $v$ es adyacente a todos los vértices de $C_{q}-\{k\}$. Luego, $C_{q}-\{k\} \in \mathcal{C}(G-k)$.

La siguiente definición será usada en la prueba del Teorema 4.4 el cual establece que cualquier grafo VPT minimal no- $[h, 2,1]$ es split y no tiene vértices estables dominados.

Definición 4.3 Una representación VPT canónica de $G$ es un par $\langle\mathcal{P}, T\rangle$ donde $T$ es un árbol cuyos vértices son los miembros de $\mathcal{C}(G), \mathcal{P}$ es la familia $\left(P_{v}\right)_{v \in V(G)}$ con $P_{v}=\{C \in \mathcal{C}(G) \mid v \in C\}$ y $P_{v}$ induce un subcamino de $T$ para todo $v \in V(G)$.

En [31] fue probado que todo grafo VPT admite una representación VPT canónica.

Teorema 4.4 Sea $G$ un grafo VPT y $h \geq 3$. Si $G$ es un grafo minimal no- $[h, 2,1]$, entonces $G \in$ Split sin vértices estables dominados.

Demostración: Caso (1): Supongamos que $G \in$ Split con partición split $(S, K)$, y $G$ tiene vértices estables dominados. Sea $\langle\mathcal{P}, T\rangle$ una VPT representación canónica de $G$, y sea $q \in V(T)$ tal que $K=C_{q}$. Asumamos que $N_{T}(q)=\left\{q_{1}, q_{2}, . ., q_{k}\right\}$, con $k>h$, y llamemos $T_{1}, T_{2}, \ldots, T_{k}$ a las ramas de $T$ 
en $q$ conteniendo a los vértices $q_{1}, q_{2}, . ., q_{k}$ respectivamente. Es claro que para cada $q_{i}$, con $1 \leq i \leq k$, existe $P_{w_{i}} \in \mathcal{P}$ tal que $q_{i} \in V\left(P_{w_{i}}\right)$ y $q \notin V\left(P_{w_{i}}\right)$. Notar que todo $w_{i} \in S$.

Supongamos que $S=\left\{w_{1}, w_{2}, . ., w_{k}\right\}$. Dado que $G$ tiene vértices estables dominados, por el item 4. del Teorema 4.3 podemos asumir, sin pérdida de generalidad, que $N\left(w_{1}\right) \varsubsetneqq N\left(w_{2}\right)$. Esto significa que $w_{1}$ y $w_{2}$ no son adyacentes en $B\left(G / C_{q}\right)$; esto es, por el item 3. del Teorema 4.3, $N_{B\left(G / C_{q}\right)}\left(w_{1}\right) \nsubseteq$ $N_{B\left(G / C_{q}\right)}\left(w_{2}\right)$. En consecuencia, existe $l \in V\left(B\left(G / C_{q}\right)\right)-\left\{w_{1}, w_{2}\right\}$, tal que $l \in N_{B\left(G / C_{q}\right)}\left(w_{1}\right)-N_{B\left(G / C_{q}\right)}\left(w_{2}\right)$. Dado que $V\left(B\left(G / C_{q}\right)\right)=S$ (por el item 1. del Teorema 4.3 y por ser $G=(S, K))$ podemos asumir que $l=w_{3}$. Entonces, por la definición de grafo rama, existe $z \in C_{q}$ tal que $z w_{1} \in E(G)$, $z w_{3} \in E(G)$ y, dado que $N\left(w_{1}\right) \varsubsetneqq N\left(w_{2}\right), z w_{2} \in E(G)$, lo cual implica que $P_{z}$ contiene a los vértices $q_{1}, q_{2}$ y $q_{3}$. Entonces $P_{z}$ no es un camino. Lo que contradice que $\langle\mathcal{P}, T\rangle$ es una representación VPT de $G$.

Concluímos que $S^{\prime}=S-\left\{w_{1}, w_{2}, . ., w_{k}\right\} \neq \emptyset$. Sea $G^{\prime}=G-S^{\prime}$. Notar que $C_{q} \in \mathcal{C}\left(G^{\prime}\right)$ y $V\left(B\left(G^{\prime} / C_{q}\right)\right)=\left\{w_{1}, w_{2}, . ., w_{k}\right\}$. Dado que $G$ es un grafo minimal no- $[h, 2,1]$, entonces $G^{\prime} \in[h, 2,1]$ y $\chi\left(B\left(G^{\prime} / C_{q}\right)\right) \leq h$.

Afirmamos que existe una $h$-coloración de $B\left(G^{\prime} / C_{q}\right)$ tal que si existe $x \in C_{q}$ y $w_{i}, w_{j} \in\left\{w_{1}, w_{2}, . ., w_{k}\right\}$ con $x w_{i} \in E(G), x w_{j} \in E(G)$ entonces

$$
w_{i} \text { y } w_{j} \text { tienen diferentes colores en } B\left(G^{\prime} / C_{q}\right) \text {. }
$$

Pues, si $w_{i}$ y $w_{j}$ tienen el mismo color en $B\left(G^{\prime} / C_{q}\right)$ entonces $w_{i} w_{j} \notin$ $E\left(B\left(G^{\prime} / C_{q}\right)\right)$. Luego podemos asumir que $N\left(w_{i}\right) \subseteq N\left(w_{j}\right)$, dado que, por hipótesis, existe $x \in C_{q}$ tal que $x w_{i} \in E(G)$ y $x w_{j} \in E(G)$. Lo cual implica que $w_{i}$ es un vértice aislado de $B\left(G^{\prime} / C_{q}\right)$. Con lo cual, podemos cambiar 
el color de $w_{i}$ a alguno de los $h-1$ colores restantes. Este proceso puede ser hecho tantas veces como sea necesario hasta que obtengamos la deseada $h$-coloración de $B\left(G^{\prime} / C_{q}\right)$.

En consecuencia, consideramos una $h$-coloración, digamos $c^{\prime}$, de $B\left(G^{\prime} / C_{q}\right)$ satisfaciendo la condición (4.2).

Ahora, damos una $h$-coloración, digamos $c$, de $B\left(G / C_{q}\right)$ como sigue: dado $w \in V\left(B\left(G / C_{q}\right)\right)$, por el Lema 3.3, existe $1 \leq i \leq k$ tal que $P_{w}$ está contenido en $T_{i}$, definimos $c(w)=c^{\prime}\left(w_{i}\right)$. Notar que, en particular, $c\left(w_{i}\right)=c^{\prime}\left(w_{i}\right)$.

Veremos que $c$ es una coloración propia de $B\left(G / C_{q}\right)$. Esto es, tenemos que ver que si $u v \in E\left(B\left(G / C_{q}\right)\right)$ entonces $c(u) \neq c(v)$. Dado que $u v \in$ $E\left(B\left(G / C_{q}\right)\right)$, por el Lema $3.3, P_{u}$ y $P_{v}$ están en diferentes ramas de $T$ en $q$ digamos $T_{i}$ y $T_{j}$. Más aún, existe $x \in C_{q}$ tal que $x u \in E(G)$ y $x v \in E(G)$, pero esto implica que $x w_{i} \in E(G)$ y $x w_{j} \in E(G)$. Luego, dado que nuestra coloración satisface la condición $(4.2), c^{\prime}\left(w_{i}\right) \neq c^{\prime}\left(w_{j}\right)$. Con lo cual, $c(u) \neq$ $c(v)$. De ahí que, nuestra coloración es propia.

Entonces, tenemos una $h$-coloración propia de $B\left(G / C_{q}\right)$ lo cual contradice que $C_{q}$ es un clique principal de $G$. Concluímos que, si $G \in$ Split entonces $G$ no tiene vértices estables dominados.

Caso (2): Supongamos que $G \notin$ Split. Como $G$ es un grafo minimal no$[h, 2,1]$, por el Teorema $4.2, G \in[h+1,2,1]$. Sea $\langle\mathcal{P}, T\rangle$ una $(h+1,2,1)$ representación de $G$ y sea $q \in V(T)$ tal que $C_{q}$ es un clique principal de $G$. Sabemos, por el item 1. del Teorema 4.3, que $V\left(B\left(G / C_{q}\right)\right)=V(G)-C_{q}$. Dado que $G \notin$ Split existen $x, y \in V\left(B\left(G / C_{q}\right)\right)$ tal que $x y \in E(G)$.

Sea $\tilde{G}$ el grafo que tiene una $(h+1,2,1)$-representación, $\left\langle\mathcal{P}^{\prime}, T\right\rangle$, donde $\mathcal{P}^{\prime}=\left(P_{v}^{\prime}\right)_{v \in V(G)}$ tal que: 
$P_{v}^{\prime}=\left\{\begin{aligned} P_{v} & \text { si } v \in C_{q} ; \\ q_{v} & \text { si } v \in V(G)-C_{q}, \text { donde } q_{v} \text { es el vértice de } P_{v} \text { más cercano a } q .\end{aligned}\right.$

Notar que $V(\tilde{G})=V(G)$. Afirmamos que $\tilde{G}$ es un grafo split, con partición split $\left(V(G)-C_{q}, C_{q}\right)$. Pues, si $x, y \in V(G)-C_{q}$ y $x y \in E(\tilde{G})$ entonces $q_{x}=q_{y}$. Esto es, $N_{G}(x) \cap C_{q}=N_{G}(y) \cap C_{q}$ lo cual contradice el item 4. del Teorema 4.3. Luego, $\tilde{G} \in$ Split y, por el Lema $4.1, C_{q}$ es un clique principal de $\tilde{G}$.

Veremos que $B\left(G / C_{q}\right)=B\left(\tilde{G} / C_{q}\right)$, de ahí que $\tilde{G} \in[h+1,2,1]-[h, 2,1]$.

Podemos asumir, por el item 4. del Teorema 4.3, que $N_{G}(x) \cap C_{q} \subseteq$ $N_{G}(y) \cap C_{q}$, porque en otro caso $G$ contendría un 4-ciclo inducido, lo cual contradice que $G \in \mathrm{VPT}$ (ver Figura 4.1). Con lo cual, existe $w \in C_{q}$ tal que $w x \in E(G), w y \in E(G)$. Y, dado que $x y \in E(G)$ entonces $P_{x}$ y $P_{y}$ están en una misma rama de $T$ en $q$. Además, por el item 4. del Teorema 4.3, $N_{G}(x) \cap C_{q} \neq N_{G}(y) \cap C_{q}$, con lo cual existe $w^{\prime} \in C_{q}$ tal que $w^{\prime} \in(N(y) \cap$ $\left.C_{q}\right)-\left(N(x) \cap C_{q}\right)$. Luego, por la existencia de $w$ y $w^{\prime}, q_{y}$ está en el camino de $T$ entre $q$ y $q_{x}$. Esto implica que $\tilde{G}$ tiene vértices estables dominados. Ahora es fácil ver que $B\left(G / C_{q}\right)=B\left(\tilde{G} / C_{q}\right)$, de ahí que $\tilde{G} \in[h+1,2,1]-[h, 2,1]$.

Entonces, por Caso (1), $\tilde{G}$ no es un grafo minimal no- $[h, 2,1]$. Esto es, existe $v \in V(\tilde{G})$ tal que $(\tilde{G}-v) \in[h+1,2,1]$.

Si $v \in V\left(B\left(\tilde{G} / C_{q}\right)\right)$, entonces $\chi\left(B\left((\tilde{G}-v) / C_{q}\right)\right)=h+1$. Más aún, por el Lema 3.2 y como $B\left(\tilde{G} / C_{q}\right)=B\left(G / C_{q}\right)$, tenemos que $B\left((\tilde{G}-v) / C_{q}\right)=$ $B\left(\tilde{G} / C_{q}\right)-v=B\left(G / C_{q}\right)-v=B\left((G-v) / C_{q}\right)$. Luego, $\chi\left(B\left((G-v) / C_{q}\right)\right)=$ $h+1$ lo cual contradice que $G$ es un grafo minimal no- $[h, 2,1]$.

Si $v \in C_{q}$, entonces, por el item 5. del Teorema 4.3, $C_{q}-v \in \mathcal{C}(G-v)$; con lo cual $C_{q}-v \in \mathcal{C}(\tilde{G}-v)$. Esto es, $\tilde{G}-v \in$ Split con partición split 
$\left(V(G)-C_{q}, C_{q}-v\right)$. Luego, por el Lema 4.1, $C_{q}-v$ es un clique principal de $\tilde{G}-v$. En consecuencia, $\chi\left(B\left((\tilde{G}-v) /\left(C_{q}-v\right)\right)\right)=h+1$. Además, es fácil ver que $B\left((\tilde{G}-v) /\left(C_{q}-v\right)\right)=B\left((G-v) /\left(C_{q}-v\right)\right)$; esto es $\chi\left(B\left((\tilde{G}-v) /\left(C_{q}-\right.\right.\right.$ $v)))=\chi\left(B\left((G-v) /\left(C_{q}-v\right)\right)\right)=h+1$ lo cual contradice que $G$ es un grafo minimal no- $[h, 2,1]$.

Concluímos que $G \in$ Split.

En el Teorema 4.3 dimos algunas condiciones necesarias en el grafo rama con respecto al clique principal de un grafo minimal no- $[h, 2,1]$. En lo que sigue, en el Teorema 4.5, usando el hecho de que todos los grafos minimales no- $[h, 2,1]$ son split sin vértices dominados en el estable y el hecho de que el clique central de un grafo split es principal, daremos más condiciones necesarias para grafos minimales no- $[h, 2,1]$.

Teorema 4.5 Sea $G$ un grafo VPT y sea $h \geq 3$. Si $G$ es un grafo minimal no-[h,2,1] con partición split $(S, K)$ entonces:

1. para todo $k \in K,|N(k) \cap S|=2$;

2. $|E(B(G / K))|=|K|$;

3. $B(G / K)$ es $(h+1)$-crítico.

Demostración: Por el Teorema 4.4, $G \in$ Split sin vértices estables dominados. Sea $(S, K)$ la partición split de $G$. Por el Lema $4.1, K$ es un clique principal de $G$.

1. Dado que $G \in \mathrm{VPT} \cap$ Split sin vértices estables dominados, $\mid N(k) \cap$ $S \mid \leq 2$ para todo $k \in K$. Supongamos que existe $k^{\prime} \in K$ tal que $\left|N\left(k^{\prime}\right) \cap S\right|<$ 2. Veremos que para todo $k \in K$ se tiene que $|N(k) \cap S|=2$. 
Por el Teorema 4.2, $G \in[h+1,2,1]$. Sea $\langle\mathcal{P}, T\rangle$ una $(h+1,2,1)$-represención de $G$ y sea $q \in V(T)$ tal que $K=C_{q}$. Por el item 5. del Teorema 4.3, $C_{q}-\left\{k^{\prime}\right\} \in \mathcal{C}\left(G-k^{\prime}\right)$

(i) $\mathrm{Si}\left|N\left(k^{\prime}\right) \cap S\right|=0$ : Entonces $B\left(\left(G-k^{\prime}\right) /\left(C_{q}-\left\{k^{\prime}\right\}\right)\right)=B\left(G / C_{q}\right)$. Esto es, $\chi\left(B\left(\left(G-k^{\prime}\right) /\left(C_{q}-\left\{k^{\prime}\right\}\right)\right)\right)=\chi\left(B\left(G / C_{q}\right)\right)=h+1$, lo cual contradice que $G$ es un grafo minimal no- $[h, 2,1]$.

(ii) Si $\left|N\left(k^{\prime}\right) \cap S\right|=1$ : Veremos que $B\left(\left(G-k^{\prime}\right) /\left(C_{q}-\left\{k^{\prime}\right\}\right)\right)=B\left(G / C_{q}\right)$. Es claro, por el item 2. del Teorema 4.3, que $V\left(B\left(\left(G-k^{\prime}\right) /\left(C_{q}-\left\{k^{\prime}\right\}\right)\right)\right)=$ $V\left(B\left(G / C_{q}\right)\right)$ y $E\left(B\left(\left(G-k^{\prime}\right) /\left(C_{q}-\left\{k^{\prime}\right\}\right)\right)\right) \subseteq E\left(B\left(G / C_{q}\right)\right)$. Sea $u v \in E\left(B\left(G / C_{q}\right)\right)$ tal que $u v \notin E\left(B\left(\left(G-k^{\prime}\right) /\left(C_{q}-\left\{k^{\prime}\right\}\right)\right)\right)$. Como $\left.\mid N\left(k^{\prime}\right) \cap S\right) \mid=1$ podemos asumir, sin pérdida de generalidad, que $\left(N(v) \cap C_{q}\right)-\left(N(u) \cap C_{q}\right)=\left\{k^{\prime}\right\}$. Luego, tenemos que $N_{B\left(G / C_{q}\right)}(v)=\{u\}$, pues si existiera $w \neq u$ tal que $w \in N_{B\left(G / C_{q}\right)}(v)$, entonces existiría $\tilde{k} \in C_{q}$ tal que $\tilde{k} w \in E(G), \tilde{k} v \in E(G)$. Y, dado que $\left|N\left(k^{\prime}\right) \cap S\right|=1$, tenemos que $\tilde{k} \neq k^{\prime}$; esto es $\tilde{k} u \in E(G)$, pues $\left(N(v) \cap C_{q}\right)-\left(N(u) \cap C_{q}\right)=\left\{k^{\prime}\right\}$. Pero entonces $\{u, v, w\} \subseteq N(\tilde{k}) \cap S$, lo cual contradice el hecho de que $|N(k) \cap S| \leq 2$ para todo $k \in K$.

En consecuencia, $d_{B\left(G / C_{q}\right)}(v)=1$ lo cual contradice el item 3. del Teorema 4.3, el cual establece que $B\left(G / C_{q}\right)$ es $(h+1)$-vértice crítico.

2. Primero probaremos que $|E(B(G / K))| \leq|K|$. Sea $e=u v \in E(B(G / K))$. Por la definición de grafo rama, existe $k \in K$ tal que $k u \in E(G), k v \in E(G)$. Esto es, para cada $e \in E(B(G / K))$ existe $k \in K$. En consecuencia, por el item 1., $|E(B(G / K))| \leq|K|$.

Ahora veremos que $|K| \leq|E(B(G / K))|$. Sea $k \in K$. Por el item 1., $|N(k) \cap S|=2$. Supongamos que $N(k) \cap S=\{u, v\}$, luego $N(u) \cap N(v) \neq \emptyset$. Como no hay vértices estables dominados, $N(u) \nsubseteq N(v), N(v) \nsubseteq N(u)$. 
Esto es, $u v \in E(B(G / K))$. Con lo cual, para cada $k \in K$ existe $u, v \in S$ tal que $u v \in E(B(G / K))$. Observar que si $\tilde{k} \in K$ tal que $\tilde{k} \neq k$, entonces $N(\tilde{k}) \cap S \neq N(k) \cap S$. Pues si $N(\tilde{k}) \cap S=N(k) \cap S$, entonces $\tilde{k}$ y $k$ son gemelos verdaderos en $G$ lo que contradice que $G$ es un grafo minimal no- $[h, 2,1]$. De ahí que, $|K| \leq|E(B(G / K))|$.

3. Sea $e=u v$ una arista de $B(G / K)$, probaremos que $\chi(B(G / K)-e)<$ $\chi(B(G / K))$. Notar que es suficiente con mostrar que existe $k \in K$ tal que $B(G / K)-e=B((G-k) /(K-\{k\}))$, pues dado que $G$ es un grafo minimal no- $[h, 2,1]$, tenemos que $G-k \in[h, 2,1]$, y entonces $\chi(B((G-k) /(K-$ $\{k\}))) \leq h<h+1=\chi(B(G / K))$.

Sea $k$ un vértice de $K$ adyacente a $u$ y $v$. Observar que, por el item 5 . del Teorema 4.3, $K-\{k\}$ es un clique de $G-k$. Dado que todo vértice de $S$ es adyacente a al menos dos vértices de $K$, tenemos que $V(B((G-$ $k) /(K-\{k\})))=V(B(G / K)-e)$. Por otro lado, es claro que cualesquiera dos vértices adyacentes en $B((G-k) /(K-\{k\}))$ son adyacentes en $B(G / K)$. Luego, sólo nos queda ver que $E(B(G / K)-e) \subseteq E(B((G-k) /(K-\{k\})))$.

Sea $x y$ una arista de $B(G / K)-e$, y sean $k_{1}, k_{2}, k_{3}$ vértices de $K$ tal que $k_{1} x \in E(G), k_{1} y \in E(G), k_{2} x \in E() G, k_{2} y \notin E(G), k_{3} x \notin E(G)$, $k_{3} y \in E(G)$. Notar que $k_{1} \neq k$ pues todo vértice de $K$ tiene exactamente dos vecinos en $S$ y $e \neq x y$. De ahí que, si $k_{2} \neq k$ y $k_{3} \neq k$, tenemos que $x y \in E(B((G-k) /(K-\{k\})))$ y la prueba es completada. Podemos asumir, sin pérdida de generalidad, que $k_{2}=k \mathrm{y}$, dado que los únicos vecinos de $k$ en $S$ son $u$ y $v$ también, sin pérdida de generalidad, podemos asumir que $x=u$.

Dado que, por el item 3. del Teorema $4.3, B(G / K)$ es $(h+1)$-vértice crítico, tenemos que todo vértice de $B(G / K)$ tiene grado al menos $h$. Con lo 
cual existe $k_{4} \in K$ tal que $k_{4} x \in E(G)$ y $k_{4} \neq k, k_{4} \neq k_{1}$. Si $k_{4} y \in E(G)$, entonces $N\left[k_{1}\right]=N\left[k_{4}\right]=\{x, y\} \cup K$ lo cual significa que $k_{1}$ y $k_{4}$ son gemelos verdaderos en $G$, y esto contradice la minimalidad de $G$. La existencia de los vértices $k_{1}, k_{3}, k_{4}$ implica que $x y \in E(B((G-k) /(K-\{k\})))$.

\subsection{Construyendo grafos minimales no- $[\mathrm{h}, 2,1]$}

Recordemos que dado un grafo cualquiera $H$ llamamos $G_{H}$ al grafo construído a partir de $H$ utilizando el procedimiento dado en la Sección 3.3.

Teorema 4.6 Sea $h \geq 3$. El grafo $G_{H}$ es un grafo minimal no- $[h, 2,1]$ si y sólo si $H$ es $(h+1)$-crítico.

Demostración: Asumamos que $G_{H}$ es un grafo minimal no- $[h, 2,1]$. Por el item 2. del Teorema 3.6, $B\left(G_{H} / K_{H}\right)=H$. En consecuencia, por el 3. del Teorema 4.5, $H$ es $(h+1)$-crítico.

Ahora, sea $H$ un grafo $(h+1)$-crítico con $V(H)=\left\{v_{1}, v_{2}, \ldots, v_{n}\right\}$. Por el Teorema 3.6 y el Lema 4.1, $\operatorname{Max}_{C \in \mathcal{C}\left(G_{H}\right)}\left(\chi\left(B\left(G_{H} / C\right)\right)\right)=\chi\left(B\left(G_{H} / K_{H}\right)\right)$ $=\chi(H)=h+1$. Luego, por el Teorema 3.5, $G_{H} \in[h+1,2,1]-[h, 2,1]$. Veamos que $G_{H}-v \in[h, 2,1]$, para todo $v \in V\left(G_{H}\right)$. Primero, si $v=$ $v_{i} \in V(H)$, usando el Lema 3.2 y el item 2. del Teorema 3.6, $B\left(\left(G_{H}-\right.\right.$ $\left.\left.v_{i}\right) / K_{H}\right)=B\left(G_{H} / K_{H}\right)-v_{i}=H-v_{i}$. Como $H$ es $(h+1)$-vértice crítico, $\chi\left(B\left(\left(G_{H}-v_{i}\right) / K_{H}\right)\right)=h$. Luego, $G_{H}-v_{i} \in[h, 2,1]$. Segundo, si $v=v_{i j}$ donde $e=v_{i} v_{j} \in E(H)$, como una consecuencia directa de la forma en que el grafo $G_{H}$ fue obtenido de $H$, tenemos que $B\left(\left(G_{H}-v_{i j}\right) /\left(K_{H}-\left\{v_{i j}\right\}\right)\right)=H-e$. 
Entonces $\chi\left(B\left(\left(G_{H}-v_{i j}\right) /\left(K_{H}-\left\{v_{i j}\right\}\right)\right)\right)=\chi(H-e)$. Y, $\chi(H-e)=h$ pues $H$ es $(h+1)$-crítico. Con lo cual, $G_{H}-v_{i j} \in[h, 2,1]$. Dado que $H$ no tiene vértices de grado $1, G_{H}$ no tiene más vértices.

\subsection{Caracterización de los grafos minimales no- $[\mathrm{h}, 2,1]$}

En esta Sección, damos una caracterización de los grafos minimales no$[h, 2,1]$, con $h \geq 3$. El principal resultado de esta Sección es el Teorema 4.7 el cual establece que los únicos grafos VPT minimales no- $[h, 2,1]$ son los grafos $G_{H}$ construídos a partir de un grafo $(h+1)$-crítico $H$.

Además, en el Teorema 4.8, mostramos que la familia de grafos construídos a partir de grafos $(h+1)$-críticos junto con la familia de subgrafos inducidos prohibidos minimales para VPT [24, 29], es la familia de subgrafos inducidos prohibidos minimales para $[h, 2,1]$, con $h \geq 3$.

Teorema 4.7 Sea $h \geq 3$ y sea $G$ un grafo VPT. G es un grafo minimal no-[h,2,1] si y sólo si existe un grafo $(h+1)$-crítico $H$ tal que $G \simeq G_{H}$.

Demostración: La recíproca sale directamente aplicando el Teorema 4.6.

Sea $G$ un grafo minimal no- $[h, 2,1]$. Por el Teorema 4.4, sabemos que $G \in$ Split sin vértices estables dominados. Sea $(S, K)$ una partición split de $G$. Por el Teorema 4.2, $G \in[h+1,2,1]$. Sea $H=B(G / K)$. Por el item 3. del Teorema 4.5, $H$ es un grafo $(h+1)$-crítico. Veamos que $G \simeq G_{H}$. Sea $G_{H}=\left(S_{H}, K_{H}\right)$. Por el item 2. del Teorema 3.6, $B\left(G_{H} / K_{H}\right)=H$; entonces $B\left(G_{H} / K_{H}\right)=$ 
$B(G / K)$. Luego, como $V\left(B\left(G_{H} / K_{H}\right)\right)=V(B(G / K)), S_{H}=S$. Además, dado que $E\left(B\left(G_{H} / K_{H}\right)\right)=E(B(G / K))$, por el item 2. del Teorema 4.5, $\left|K_{H}\right|=|K|$ y, por el item 1. del Teorema 4.5, $|N(k) \cap S|=2$ para todo $k \in K$. Supongamos que $N(k) \cap S=\left\{v_{i}, v_{j}\right\}$; veremos que $v_{i} v_{j} \in E(H)$. Es claro que $v_{i} k \in E(G)$ y $v_{j} k \in E(G)$. Además, por el item 2. del Teorema 4.3 , existen $k^{\prime}, k^{\prime \prime} \in K$ tales que $k^{\prime} v_{i} \in E(G), k^{\prime \prime} v_{j} \in E(G)$. Si $k^{\prime}=k^{\prime \prime}$ entonces, como $|N(k) \cap S|=2$ para todo $k \in K$, tenemos que $k^{\prime}$ y $k$ son gemelos verdaderos en $G$, lo cual contradice que $G$ es un grafo minimal no$[h, 2,1]$. Luego, $k^{\prime} \neq k^{\prime \prime}$. Esto es, $k^{\prime} v_{j} \notin E(G)$ y $k^{\prime \prime} v_{i} \notin E(G)$. De ahí que, $v_{i} v_{j} \in E(H)$.

En consecuencia, podemos definir una función $f$ que asigna a cada vértice $k \in K$ una arista $v_{i} v_{j} \in E(H)$, esto es, un elemento de $K_{H}$. Notar que en $G_{H}$ el vértice $v_{i j} \in K_{H}$ es adyacente exactamente a $v_{i}$ y $v_{j}$. Luego, la función $f$ puede ser extendida a una nueva función $\tilde{f}$ desde $K \cup S$ a $K_{H} \cup S_{H}$, siendo la identidad desde $S$ a $S_{H}$. Además, $\tilde{f}$ es un isomorfismo entre $G$ y $G_{H}$.

Teorema 4.8 Sea $h \geq 3$. Un grafo $G$ es minimal no-[h,2,1] si y sólo si $G$ es uno de los miembros de $F_{0}, F_{1}, \ldots, F_{16} o G \simeq G_{H}$, donde $H$ es un grafo $(h+1)$-crítico.

Demostración: Por el Teorema 4.7, si $G \simeq G_{H}$ donde $H$ es un grafo $(h+1)$ crítico, entonces $G$ es un grafo minimal no- $[h, 2,1]$.

Si $G$ es alguno de los miembros de $F_{0}, . ., F_{16}$ entonces $G \notin$ VPT y $G-v \in$ VPT, para todo $v \in V(G)$. Además, en [9] fue probado que $G-v \in \mathrm{EPT}$, para todo $v \in V(G)$. Esto es, $G-v \in \mathrm{VPT} \cap \mathrm{EPT}=[3,2,1][20]$, lo cual implica que $G-v \in[h, 2,1]$. En consecuencia, $G$ es un grafo minimal no- $[h, 2,1]$. 
Sea $h \geq 3$ y sea $G$ un grafo minimal no- $[h, 2,1]$.

Caso (1): $G \notin \mathrm{VPT}$. Como $G$ es un grafo minimal no- $[h, 2,1]$, entonces $G-v \in[h, 2,1]$ para todo $v \in V(G)$. Luego, $G-v \in$ VPT para todo $v \in V(G)$. Entonces, $G$ es un subgrafo inducido prohibido minimal de VPT. En consecuencia, $G$ es uno de los miembros de $F_{0}, F_{1}, \ldots, F_{16}$.

Caso (2): $G \in$ VPT. Entonces, por el Teorema 4.7, $G \simeq G_{H}$, donde $H$ es un grafo $(h+1)$-crítico.

Notar que, como todo $G_{H}$ es un grafo VPT, ningún miembro de $F_{0}, F_{1}, \ldots$, $F_{16}$ es un subgrafo inducido de $G_{H}$. 


\section{Capítulo 5}

\section{Una condición necesaria para}

grafos EPT y una nueva familia de subgrafos inducidos prohibidos minimales

Un camino en un árbol puede ser dado tanto por su conjunto de vértices como por su conjunto de aristas. En este último caso, si $G$ es el grafo de intersección de una familia de caminos en un árbol, es decir, los vértices de $G$ corresponden a los caminos, y dos vértices de $G$ son adyacentes en $G$ si y sólo si los caminos correspondientes comparten al menos una arista, decimos que $G$ es el grafo arista-intersección de estos caminos y lo denotamos por $G=E P T(P)$, donde $P$ es una familia de caminos en un árbol huésped $T$.

Decimos que $G$ es EPT si es el grafo arista-intersección de una familia de caminos en un árbol. 
Observamos que, por ser una clase de grafos de intersección, la clase de grafos EPT es hereditaria, es decir, si un grafo $G$ es EPT entonces todo subgrafo inducido $H$ de $G$ también es EPT. De hecho, si $G=E P T(P)$ para formar una representación EPT de $H$, basta con tomar los caminos de $P$ que corresponden a vértices de $H$ y el mismo árbol $T$.

Los grafos EPT fueron introducidos por Syslo, en 1978, donde fueron denominados los grafos ciclo fundamental [34]. Su estudio fue motivado por encontrar el mayor conjunto posible de aristas en un árbol generador de un grafo de modo que los ciclos formados por dichas aristas no se intersecten. Además, en [34], Syslo conjeturó que EPT $\cap$ Cordal $=\mathrm{EPT} \cap$ VPT. Una prueba de esta conjetura fue presentada por él mismo en [35] en el año 1985 usando los resultados estructurales obtenidos por Golumbic y Jamison en [15], en particular las caracterizaciones de los grafos que son simultáneamente VPT y EPT.

Golumbic y Jamison presentaron varios resultados para la clase EPT [16]. Estos resultados son referentes a los cliques, a la estructura de los grafos y al comportamiento de la clase para algunos problemas en grafos en relación con la Conjetura Fuerte de los Grafos Perfectos. Los mismos autores, en [15], probaron que el problema de reconocimiento de los grafos EPT es NPcompleto. Tarjan consideró la descomposición por cliques de estos grafos en [36].

Finalmente, en 1986, Monma y Wei publicaron el trabajo más completo, hasta entonces, sobre las clases de grafos de intersección de caminos en un árbol donde estandarizaron tanto la terminología como el enfoque para el estudio de estas clases [31]. 
Si consideramos la intersección por vértices de subárboles de un árbol, tenemos exactamente la clase de los grafos cordales. Una pregunta natural es el análisis de la intersección por aristas de subárboles de un árbol. Otro caso a analizar es el de la intersección de una familia de caminos en un grafo cualquiera, sea ésta en aristas o en vértices. Golumbic y Jamison mostraron que, en los casos citados, no se crea ninguna nueva clase y que los grafos en cuestión son todos los grafos [16]. Como consecuencia del análisis hecho para obtener estos resultados, es posible también mostrar que todo grafo es un grafo de intersección por aristas de subestrellas de una estrella.

Los resultados presentados en este Capítulo fueron publicados en [1].

En este Capítulo, damos una nueva condición necesaria para ser un grafo EPT. Para esto nos basamos en la estructura de los cliques de un grafo EPT. Además, encontramos una nueva familia de subgrafos inducidos prohibidos minimales para la clase EPT.

\subsection{Resultados básicos sobre cliques en gra- fos EPT}

En los grafos EPT los cliques tienen una estructura particular (ver [16]) la cual es muy importante para nuestro resultado.

El grafo garra $K_{1,3}$ consiste de un vértice central y tres aristas incidentes en él. Estas aristas son llamadas las patas de la garra (ver Figura 5.1). 


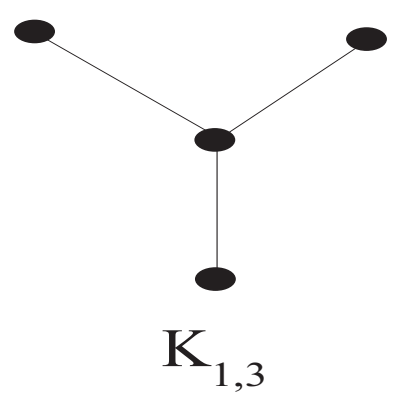

Figura 5.1: Grafo garra.

Sea $\langle P, T\rangle$ una representación EPT de $G$. Para cualquier arista $e$ de $T$, sea $\mathrm{P}[e]=\{\mathrm{P} \in P \mid e$ es una arista de $\mathrm{P}\}$. Para cualquier garra $K_{1,3}$ en $T$, sea $\mathrm{P}\left[K_{1,3}\right]=\left\{\mathrm{P} \in P \mid \mathrm{P}\right.$ contiene dos patas de $\left.K_{1,3}\right\}$. La colección $\mathrm{P}[e]$ corresponde a un clique en $G$ y es llamada un clique arista. Similarmente, $\mathrm{P}\left[K_{1,3}\right]$ también corresponde a un clique en $G$ y es llamada un clique garra. (Ver un ejemplo en la Figura 5.2).

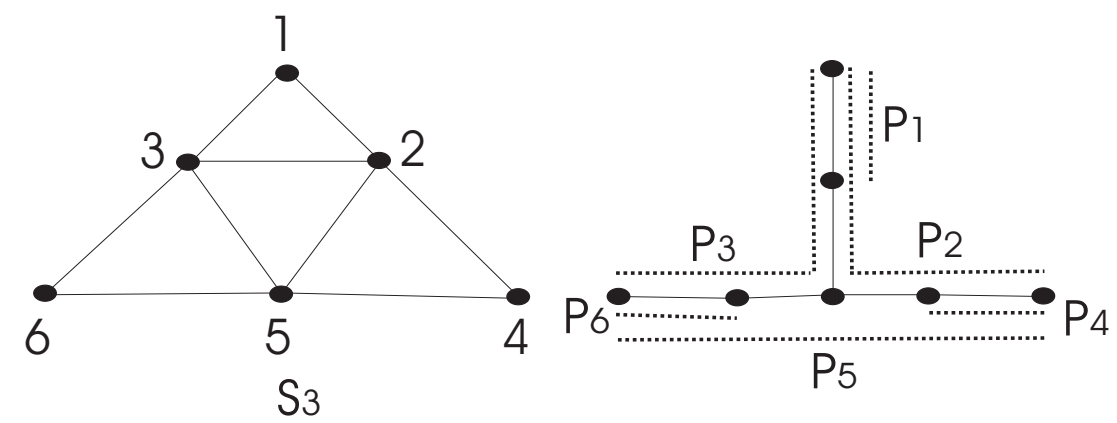

Figura 5.2: Una representación EPT del sol $S_{3}$. El triángulo central $\{2,3,5\}$ es un clique garra; los otros tres triángulos son cliques aristas.

Teorema 5.1 [16] Sea $G$ un grafo y $\langle P, T\rangle$ una representación EPT de $G$. Cualquier clique de $G$ corresponde o a una subcolección de caminos de la 
forma $P[e]$ para alguna arista e en $T$ o de la forma $P\left[K_{1,3}\right]$ para alguna garra $K_{1,3}$ en $T$.

\subsection{Una condición necesaria para grafos EPT}

En esta sección damos una nueva condición necesaria para ser un grafo EPT basada en los siguientes conceptos:

Definición 5.1 Sea $C \in \mathcal{C}(G)$. Un vértice $v$ de $G$ es un satélite de $\boldsymbol{C}$ si $v \notin C$ y $B_{v}=N(v) \cap C$ es un subconjunto propio no vacío de $C$.

El conjunto $B_{v}$ es llamado la base de $\boldsymbol{v}$ y éste se dice minimal si ninguna otra base de un satélite de $C$ está propiamente contenida en $B_{v}$.

Teorema 5.2 Sea $G$ un grafo EPT y sea $C$ un clique de $G$. Si $w \in C$ entonces $w$ pertenece a lo sumo a dos bases minimales diferentes de satélites no adyacentes de $C$.

Demostración: Sea $\langle P, T\rangle$ una representación EPT de $G$. Por el Teorema 5.1 sabemos que $C$ es un clique arista o un clique garra. Consideramos las dos posibilidades:

Caso (1): $C=\left\{v_{1}, \ldots, v_{k}\right\}$ es un clique arista, esto es, cada camino $P_{v_{i}}$, con $1 \leq i \leq k$, representando vértices de $C$ en $\langle P, T\rangle$, tiene una arista $e=x y$ de $T$ en común.

Sea $w \in C$, supongamos por el absurdo, que $w$ está en tres bases minimales diferentes de satélites no adyacentes de $C$, digamos $y_{1}, y_{2}, y_{3}$. 
Sea $P_{w}$ el camino correspondiente al vértice $w$ en $\langle P, T\rangle$, entonces $\mid V\left(P_{w}\right) \cap$ $V\left(P_{y_{i}}\right) \mid \geq 2$, para $i=1,2,3$. Dado que $P_{w}$ es un camino que pasa por la arista e y $w$ es adyacente a $y_{i}$, para $i=1,2,3$, tenemos que al menos dos de los caminos $P_{y_{i}}, 1 \leq i \leq 3$, deben estar en una misma componente conexa del grafo $(T-e)$. Supongamos que $P_{y_{1}}$ y $P_{y_{2}}$ son esos caminos. Dado que $y_{1}$ e $y_{2}$ son ambos adyacentes a $w$ tenemos que los caminos $P_{y_{1}}$ y $P_{y_{2}}$ deben tener al menos una arista en común con el camino $P_{w}$, y esta arista no puede ser la misma para ambos caminos dado que $y_{1}$ no es adyacente a $y_{2}$. Sean $e_{1}$, $e_{2}$ esas aristas. Es claro que $e_{1} \neq e$ y $e_{2} \neq e$, porque $y_{1} \notin C$ e $y_{2} \notin C$, por definición de satélite de $C$. Entonces, dado que $P_{w}$ es un camino estas aristas $e_{1}, e_{2}$ estarán en un mismo camino $\mathrm{P}$ de $(T-e)$. Supongamos que $e_{1}$ está más cercana a $e$ que $e_{2}$ en este camino $\mathrm{P}$, entonces cada camino $P_{v_{i}}$, con $v_{i} \in C$, que pasa por $e_{2}$ pasará por $e_{1}$ también, esto es, cada camino $P_{v_{i}}$, con $v_{i} \in C$, que intersecta a $P_{y_{2}}$ en al menos una arista, intersectará a $P_{y_{1}}$ en al menos una arista también, lo cual contradice la minimalidad de las bases.

Caso (2): $C=\left\{v_{1}, \ldots, v_{k}\right\}$ es un clique garra en el cual la garra está formada por las aristas $e_{1}, e_{2}, e_{3} \mathrm{y}$ un vértice central $z$.

Entonces todo camino $P_{v_{i}}, 1 \leq i \leq k$, tiene exactamente dos de las aristas $e_{i}$, con $i=1,2,3$, dado que ellos son caminos que se tocan en al menos dos vértices del árbol huésped $T$. Sea $w \in C$, supongamos, por el absurdo, que $w$ está en tres bases minimales diferentes de satélites no adyacentes de $C$, digamos $y_{1}, y_{2}, y_{3}$. Sea $P_{w}$ el camino que representa a $w$ en $\langle P, T\rangle$.

Observemos que $(T-z)$ es un grafo que tiene tres componentes conexas, digamos $C_{1}, C_{2}$ y $C_{3}$. Si los caminos $P_{y_{i}}$ correspondientes a los satélites $y_{i}$, $1 \leq i \leq 3$, intersectan más de una componente conexa de $(T-\{z\})$ estamos 
en el Caso (1). Entonces cada $P_{y_{i}}, 1 \leq i \leq 3$, intersecta exactamente una componente conexa de $(T-\{z\})$. Supongamos que $P_{y_{i}}$ intersecta $C_{i}$, para $i=1,2,3$. Dado que $w$ es adyacente a $y_{i}, 1 \leq i \leq 3$, tenemos que el camino $P_{w}$ debe tener al menos una arista en común con cada camino $P_{y_{i}}, 1 \leq i \leq 3$. Pero dado que los caminos $P_{y_{i}}$ intersectan sólo a la componente conexa $C_{i}$, para cada $1 \leq i \leq 3, P_{w}$ tendría a $e_{1}, e_{2}$ y $e_{3}$ como aristas, lo cual contradice el hecho de que $P_{w}$ es un camino.

Cabe destacar que el Teorema 5.2 da una condición necesaria para ser un grafo EPT, pero no una condición suficiente. Por ejemplo la familia de los grafos $A_{n}$, con $n \geq 4$, es una familia de grafos que no son EPT y que, sin embargo, verifican que si $C$ es un clique de $A_{n}$ y $w \in C$ entonces $w$ pertenece a lo sumo a dos bases minimales diferentes de satélites no adyacentes de $C$ (ver $[19])$.

\subsection{Nuevos subgrafos prohibidos minimales para la clase EPT}

En esta Sección analizaremos si con el resultado del Teorema 5.2 podrían aparecer nuevos grafos prohibidos minimales para la clase EPT (ver [19]). Más claramente, veremos que el resultado dado en el Teorema 5.2 conduce a exactamente tres nuevos subgrafos inducidos prohibidos minimales para la clase EPT. (Ver Figura 5.7).

Supongamos que $C$ es un clique, $y_{1}, y_{2}, y_{3}$ son tres satélites de $C$ y $B_{1}, B_{2}$, $B_{3}$ son bases de $y_{1}, y_{2}$ e $y_{3}$, respectivamente. Dado que estamos interesados 
en configuraciones minimales que no satisfagan las condiciones del Teorema 5.2 estas bases deben tener las siguientes propiedades:

1. Existe un único vértice de $C$, digamos 1 , tal que $1 \in B_{i}$, para $i=1,2,3$,

2. $B_{i} \nsubseteq B_{j}$, para $i \neq j, i=1,2,3, j=1,2,3$,

3. $\bigcup B_{i}=C$,

4. $B_{i} \subsetneq C$, para $i=1,2,3$.

Caso (1): Supongamos que los vértices de $C$, diferentes de 1 , pertenecen a lo sumo a una base. Notar que debe haber exactamente un vértice, diferente de 1, perteneciendo a cada base, por 2. y la minimalidad. Supongamos que $2 \in B_{1}, 3 \in B_{2}$ y $4 \in B_{3}$ (Ver Figura 5.3).

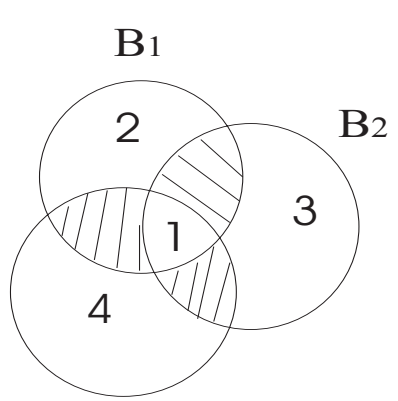

B3

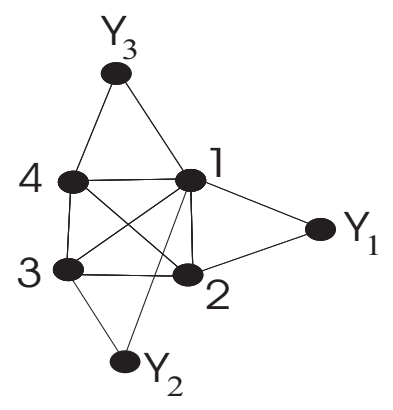

$\mathrm{S}_{1}$

Figura 5.3: Los vértices de $C$, diferentes de 1, pertenecen a lo sumo a una base, y el grafo obtenido en este caso.

Caso (2): Supongamos que exactamente un vértice de $C$, diferente de 1 , pertenece a dos bases minimales distintas, digamos $2 \in B_{1} \cap B_{2}$. Dado que las bases son minimales y diferentes debe haber al menos un vértice de $C$ 
perteneciendo sólo a $B_{2}$, digamos $3 \in B_{2}$. Ahora, debe existir un vértice de $C$ perteneciendo sólo a $B_{1}$, porque en otro caso tendríamos que $B_{1} \subseteq B_{2}$, supongamos que $4 \in B_{1}$. Por el mismo motivo, debe existir un vértice de $C$ perteneciendo sólo a $B_{3}$, digamos $5 \in B_{3}$, en otro caso tendríamos que $B_{3} \subseteq B_{1}$ o $B_{3} \subseteq B_{2}$. Pero notemos que en este caso si removemos el vértice 2 retornamos al caso anterior. Entonces el grafo $G$ obtenido tiene $S_{1}$ como subgrafo inducido (Ver Figura 5.4).

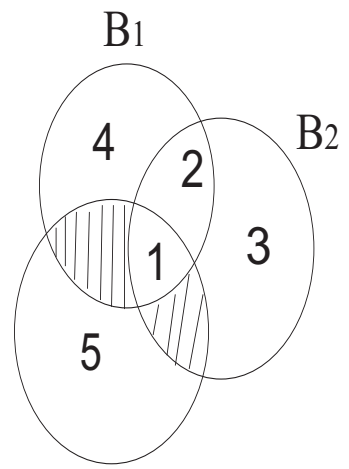

B3

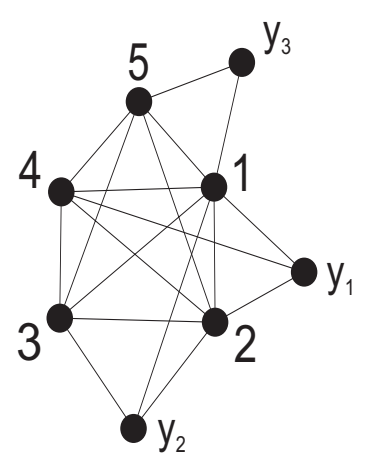

G

Figura 5.4: Exactamente un vértice de $C$, diferente de 1, pertenece a dos bases minimales diferentes.

Caso (3): Supongamos que exactamente dos vértices de $C$, diferentes de 1 , pertenecen a dos bases minimales diferentes, supongamos que $2 \in B_{1} \cap B_{2}, 3 \in$ $B_{1} \cap B_{3}$. Ahora vemos que debe haber al menos un vértice de $C$ perteneciendo sólo a $B_{2}$, porque en otro caso tendríamos que $B_{2} \subseteq B_{1}$, supongamos que $4 \in B_{2}$. Y debe haber al menos un vértice perteneciendo sólo a $B_{3}$, en otro caso tendríamos que $B_{3} \subseteq B_{1}$, supongamos que $5 \in B_{3}$ (Ver Figura 5.5). 


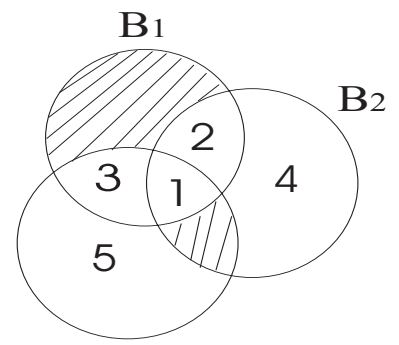

$\mathrm{B} 3$

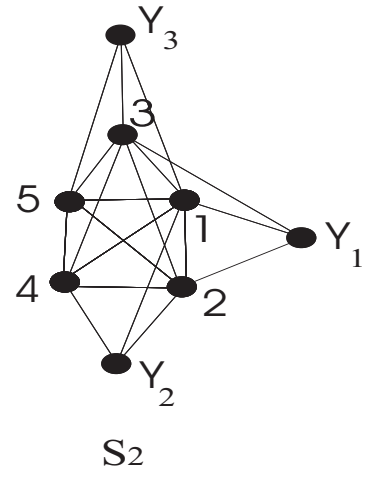

$\mathrm{S} 2$

Figura 5.5: Exactamente dos vértices de $C$, diferentes de 1, pertenecen a dos bases minimales diferentes, y el grafo obtenido en este caso.

Caso (4): Supongamos que exactamente tres vértices de $C$, diferentes de 1 , pertenecen a dos bases minimales diferentes, digamos $2 \in B_{1} \cap B_{2}$, $3 \in B_{2} \cap B_{3}, 4 \in B_{1} \cap B_{3}$. Notemos que no es necesario agregar ningún otro vértice a las bases por las condiciones de minimalidad. (Ver Figura 5.6).

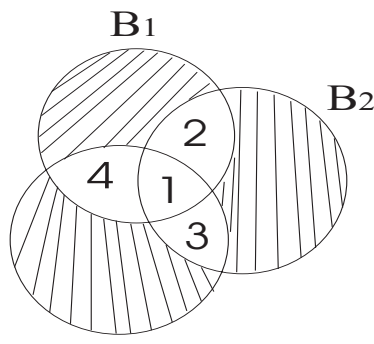

B3

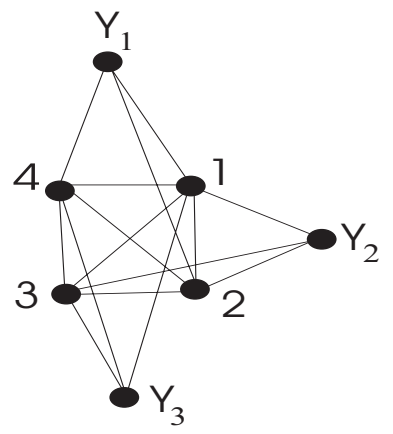

$\mathrm{S}_{3}$

Figura 5.6: Exactamente tres vértices de $C$, diferentes de 1, pertenecen a dos bases minimales diferentes, y el grafo obtenido en este caso.

Es fácil de ver que los subgrafos obtenidos removiendo cualquier vértice 
de $S_{1}, S_{2}$ o $S_{3}$ son grafos EPT, esto es, todo subgrafo inducido de $S_{1}, S_{2}$ o $S_{3}$ es un grafo EPT.

Entonces tenemos el siguiente teorema:

Teorema $5.3 S_{1}, S_{2}, S_{3}$ son subgrafos inducidos prohibidos minimales para la clase EPT.

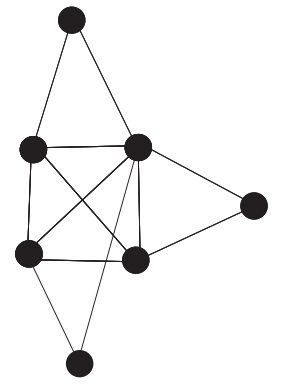

$\mathrm{S} 1$

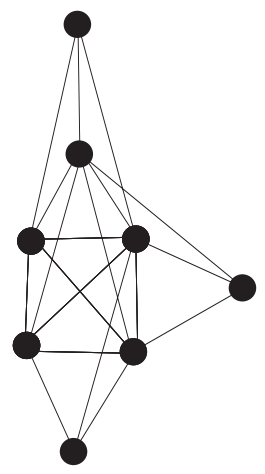

$\mathrm{S}_{2}$

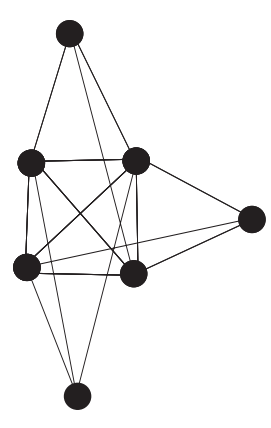

$\mathrm{S} 3$

Figura 5.7: Una nueva familia de subgrafos inducidos prohibidos minimales para la clase EPT. 


\section{Capítulo 6}

\section{Grafos EPT en árboles de grado acotado}

En este Capítulo nos enfocamos en los grafos EPT que pueden ser representados en un árbol con grado acotado. Respondemos negativamente una pregunta que Golumbic, Lypshteyn y Stern dejaron abierta en [19], para esto nos basamos en la representación EPT que tienen los ciclos de un grafo EPT.

Los resultados presentados en este Capítulo están incluídos en un trabajo que se encuentra en prensa [2].

\subsection{Resultados conocidos}

Definición 6.1 [19] Sea $\langle\mathcal{P}, T\rangle$ una representación EPT de un grafo G. Una torta de tamaño $\boldsymbol{n}$ es un subgrafo estrella de $T$ con vértice central $q y$ vecinos $q_{1}, \ldots, q_{n}$ tal que cada "porción" $q_{i} q q_{i+1}$ para $1 \leq i \leq n$ está contenida en un miembro diferente de $\mathcal{P}$; asumimos que la adición es módulo $n$. (Ver 
Figura 6.1).

Sea $\langle\mathcal{P}, T\rangle$ una representación EPT de un grafo $G$. Se sabe que los ciclos sin cuerdas de $n$ vértices, denotados por $C_{n}$, son grafos EPT. Además, en [19] fue probado que si $G$ tiene un ciclo sin cuerdas de longitud $n \geq 4$, entonces $\langle\mathcal{P}, T\rangle$ contiene una torta de tamaño $n$.
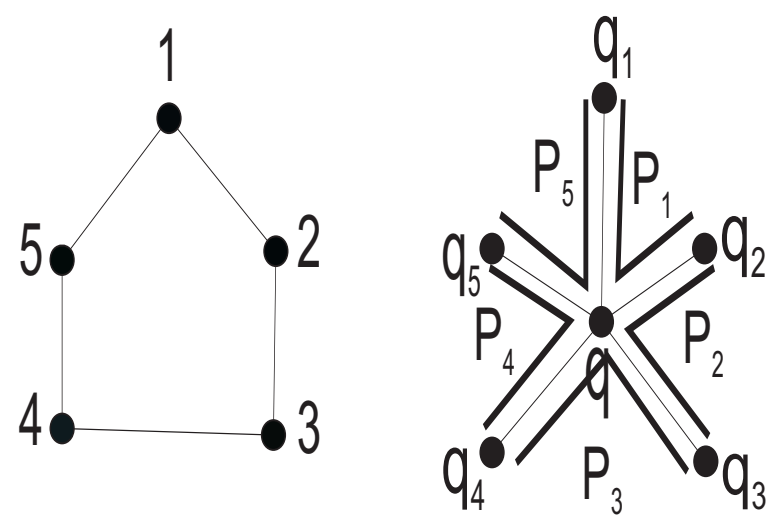

Figura 6.1: El ciclo $C_{5}$ y una representación EPT: una torta de tamaño 5.

Notemos que, en una torta, los caminos correspondientes a $k$ vértices consecutivos del ciclo cubren $k+1$ aristas incidentes en $q$.

Dado que una torta de tamaño $n$ para un ciclo $C_{n}$ de tamaño $n$ requiere un árbol huésped con un vértice de grado $n$, tenemos el siguiente teorema.

Teorema 6.1 [16] Sea $G \in E P T$ y $h \geq 3$. Si $G \in[h, 2,2]$, entonces $G$ es libre de $\left\{C_{n} \mid n>h\right\}$.

En [19], Golumbic, Lipshteyn y Stern estudiaron varios aspectos de las clases $[h, 2,2]$, para $h \geq 2$. En particular probaron que en la clase de los grafos EPT, la recíproca del Teorema 6.1 es verdadera para $h=3$ y falsa 
para $h=4$. Usaron resultados generales sobre los grafos Cordales y los grafos Débilmente Cordales (recordemos que un grafo es Débilmente Cordal si no contiene ciclos inducidos $C_{n}$, con $n \geq 5$, ni sus complementos como subgrafos inducidos) los cuales se aplican sólo cuando $h=3$ o $h=4$. Su prueba es basada en los hechos de que $[3,2,2]=\operatorname{EPT} \cap$ Cordal y $[4,2,2]=\operatorname{EPT} \cap$ Débilmente Cordal [20]. Dejaron abierta la pregunta de si la recíproca del Teorema 6.1 es verdadera para $h \geq 5$.

En este trabajo, se responde negativamente a esta pregunta. Describimos, para cada $h \geq 5$, un grafo EPT libre de $\left\{C_{n} \mid n>h\right\}$ y que sin embargo no se puede representar en un árbol huésped con grado máximo $h$.

\subsection{Nuestros resultados}

En lo que sigue presentamos los grafos que serán usados para responder la pregunta hecha por Golumbic et al. en [19]: Puede cualquier grafo EPT sin ciclos inducidos de tamaño más grande que $h$ ser representado en un árbol huésped con grado máximo $h$ ?

Definición 6.2 Sean $n_{1}, n_{2}$ y $n_{3}$ enteros positivos. Un prisma general $F_{n_{1}, n_{2}, n_{3}}$ consiste de dos triángulos $\left\{a_{1}, a_{2}, a_{3}\right\}$ y $\left\{b_{1}, b_{2}, b_{3}\right\}$; y tres caminos disjuntos sin cuerdas $Q_{i}$ para $1 \leq i \leq 3$ de longitud (número de aristas) $n_{i}$

y vértices extremos $a_{i}, b_{i}$ respectivamente. (En la Figura 6.2 se muestra un ejemplo ). 


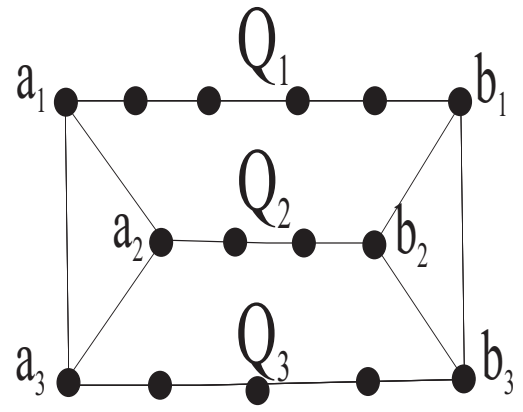

Figura 6.2: El prisma general $F_{5,3,4}$.

Observar que:

(1) El prisma general $F_{1,1,1}$ es isomorfo al complemento del grafo $C_{6}$.

(2) Por simetría, en un prisma general, el orden de los subíndices es irrelevante, esto es, $F_{n_{1}, n_{2}, n_{3}} \simeq F_{n_{1}, n_{3}, n_{2}} \simeq F_{n_{2}, n_{1}, n_{3}} \simeq F_{n_{2}, n_{3}, n_{1}} \simeq F_{n_{3}, n_{2}, n_{1}} \simeq$ $F_{n_{3}, n_{1}, n_{2}}$.

(3) El prisma general $F_{n_{1}, n_{2}, n_{3}}$ tiene $n_{1}+n_{2}+n_{3}+3$ vértices.

(4) El prisma general $F_{n_{1}, n_{2}, n_{3}}$ sin los vértices $a_{1}$ y $b_{3}$ es un camino sin cuerdas de longitud $n_{1}+n_{2}+n_{3}$.

(5) El prisma general $F_{n_{1}, n_{2}, n_{3}}$ contiene exactamente tres ciclos inducidos:

$$
\begin{array}{lll}
Q_{1} \widehat{Q_{2}} & \text { de longitud } & n_{1}+n_{2}+2 \\
Q_{1} \widehat{Q_{3}} & \text { de longitud } & n_{1}+n_{3}+2 \\
Q_{2} \widehat{Q_{3}} & \text { de longitud } & n_{2}+n_{3}+2
\end{array}
$$

donde $Q_{i} \widehat{Q_{j}}$ es la concatenación de los caminos $Q_{i}$ y la reversa de $Q_{j}$.

Lema 6.1 El prisma general $F_{n_{1}, n_{2}, n_{3}}$ es un $[h, 2,2]$ grafo para $h=n_{1}+n_{2}+$ $n_{3}+2$. 
Demostración: Sea $T$ una estrella con vértice central $q$ y vértices pendientes $q_{i}$ para $1 \leq i \leq n_{1}+n_{2}+n_{3}+2$. Para cada $i, 1 \leq i \leq n_{1}+n_{2}+n_{3}+1$, sea $P_{i}$ el subcamino del árbol huésped $T$ inducido por los vértices $q_{i}, q$ y $q_{i+1}$.

El grafo arista-intersección de esta familia de caminos es un camino sin cuerdas isomorfo a $F_{n_{1}, n_{2}, n_{3}} \sin$ los vértices $a_{1}$ y $b_{3}$; esto es, necesitamos agregar a esta familia otros dos caminos, digamos $P$ y $P^{\prime}$, representando a los vértices $a_{1}$ y $b_{3}$ respectivamente.

Sea $P$ el subcamino de $T$ inducido por los vértices $q_{1}, q$ y $q_{n_{1}+n_{2}+2} ;$ y sea $P^{\prime}$ el subcamino de $T$ inducido por los vértices $q_{n_{1}+n_{2}+n_{3}+2}, q$ y $q_{n_{1}+1}$. Ver Figura 6.3.

Es claro que el vértice representado por $P$ es adyacente a aquellos representados por $P_{1}, P_{n_{1}+n_{2}+1}$ y $P_{n_{1}+n_{2}+2}$; mientras que el vértice representado por $P^{\prime}$ es adyacente a aquellos representados por $P_{n_{1}+n_{2}+n_{3}+1}, P_{n_{1}} \mathrm{y}$ $P_{n_{1}+1}$; de esta manera obtenemos un $(h, 2,2)$-representacoón del prisma general $F_{n_{1}, n_{2}, n_{3}}$.
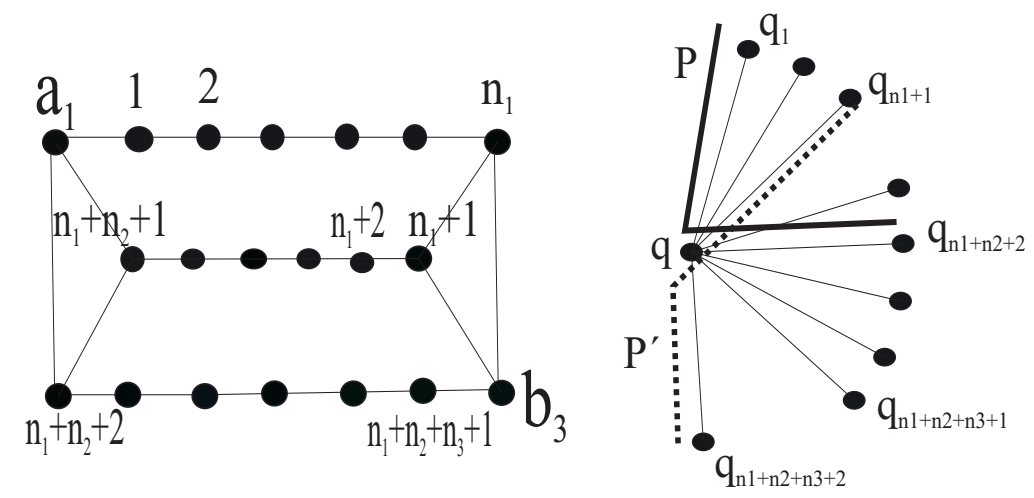

Figura 6.3: El prisma general $F_{n_{1}, n_{2}, n_{3}}$ y una $(h, 2,2)$-representación, donde $h=n_{1}+n_{2}+n_{3}+2$. 
El siguiente teorema muestra que los ciclos inducidos no son las únicas estructuras en un grafo EPT que imponen condiciones en los grados de los vértices del árbol huésped.

Teorema 6.2 Sea $h=n_{1}+n_{2}+n_{3}+1$. El prisma general $F_{n_{1}, n_{2}, n_{3}}$ no es un $[h, 2,2]$ grafo, sin embargo es libre de $\left\{C_{n} \mid n>h\right\}$.

Demostración: El caso $n_{1}=n_{2}=n_{3}=1$ fue resuelto por Golumbic et al. en [19] como mencionamos en la Sección 6.1. Luego, asumimos $n_{1} \geq 2$, lo cual significa que $Q_{1}$ tiene al menos tres vértices.

Supongamos que $F_{n_{1}, n_{2}, n_{3}} \in[h, 2,2]$ para $h=n_{1}+n_{2}+n_{3}+1$; y sea $\langle\mathcal{P}, T\rangle$ una representación EPT satisfaciendo que el grado de todo vértice de $T$ es menor o igual que $h$.

Dado que $Q_{1} \widehat{Q_{2}}$ induce un ciclo de tamaño $n_{1}+n_{2}+2$, en la representación hay una torta de tamaño $n_{1}+n_{2}+2$. Llamamos $q$ al vértice central de esta torta.

Por otro lado, dado que $Q_{1} \widehat{Q_{3}}$ también induce un ciclo, en la representación hay otra torta. Afirmamos que el vértice central de la segunda torta es el mismo vértice $q$. Esto se sigue de que:

(i) en una torta, la intersección por vértices de tres caminos correspondientes a tres vértices consecutivos es exactamente el vértice central de la torta;

(ii) los primeros tres vértices de $Q_{1}$ son consecutivos en ambos ciclos $Q_{1} \widehat{Q_{2}}$ y $Q_{1} \widehat{Q_{3}}$.

Ahora, los caminos correspondientes a los vértices de $Q_{1} \widehat{Q_{2}}$ cubren $n_{1}+$ $n_{2}+2$ aristas de $T$ incidentes en $q$. 
Por otro lado, los caminos correspondientes a los $n_{3}-1$ vértices internos de $Q_{3}$ deben cubrir $n_{3}$ aristas de $T$ incidentes en $q$ las cuales son diferentes de las $n_{1}+n_{2}+2$ cubiertas por los vértices de $Q_{1} \widehat{Q_{2}}$, porque ningún vértice interno de $Q_{3}$ es adyacente a un vértice de $Q_{1} \widehat{Q_{2}}$.

Obtenemos que $q$ tiene grado al menos $n_{3}+\left(n_{1}+n_{2}+2\right)$, lo cual contradice la asunsión de que cada vértice de $T$ tiene grado a lo sumo $h=n_{1}+n_{2}+n_{3}+1$.

Finalmente, por (5) y dado que $n_{1}+n_{2}+2 \leq n_{1}+n_{2}+n_{3}+1$; $n_{1}+n_{3}+2 \leq n_{1}+n_{2}+n_{3}+1$ y $n_{2}+n_{3}+2 \leq n_{1}+n_{2}+n_{3}+1$, el prisma general $F_{n_{1}, n_{2}, n_{3}}$ no tiene ciclos de tamaño más grande que $h=n_{1}+n_{2}+n_{3}+1$.

Finalmente, damos el siguiente teorema el cual generaliza el Teorema 6.1:

Teorema 6.3 Sea $h \geq 4$. Si $G \in[h, 2,2]$, entonces $G$ es libre de $\left\{F_{n_{1}, n_{2}, n_{3}}, C_{n} \mid n>\right.$ hy $\left.n_{1}+n_{2}+n_{3}+1=h\right\}$. 


\section{Capítulo 7}

\section{Conclusiones y trabajo futuro}

- En el Capítulo 3, damos, para cualquier $h \geq 3$, una caracterización de los grafos $[h, 2,1]$, y probamos que reconocer los grafos que pertenecen a esta clase es un problema NP-completo. Además, mostramos una familia, llamada SVS, en la cual este problema es polinomial.

Estamos trabajando en describir una subclase más grande de VPT donde este problema permanezca polinomial. Por otro lado, estamos analizando la posibilidad de extender las técnicas usadas en este capítulo para caracterizar las clases $[h, 2,2]$.

- En el Capítulo 4, mostramos que todos los grafos VPT que son minimales no- $[h, 2,1]$, con $h \geq 3$, son split sin vértices estables dominados (ver Figura 7.1). Usando este resultado, caracterizamos la clase $[h, 2,1]$ por subgrafos inducidos prohibidos minimales para cada $h \geq 3$ fijo. 


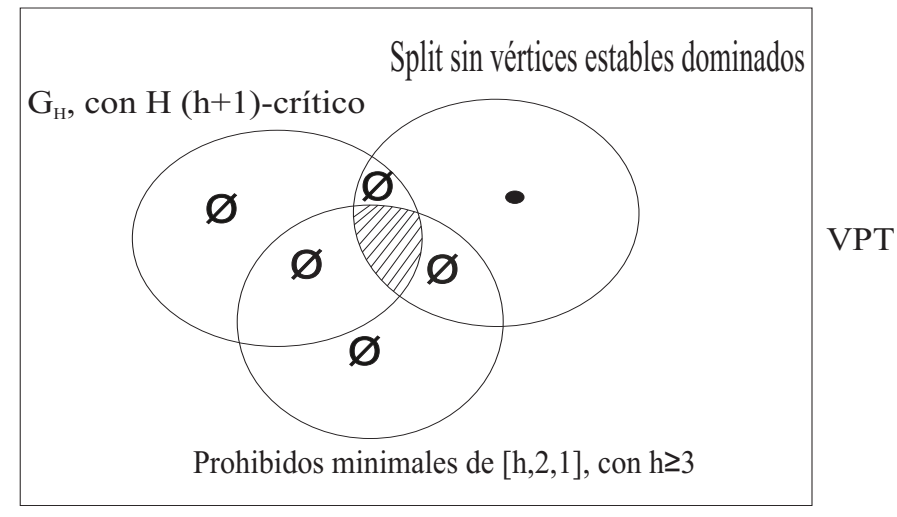

Figura 7.1: Todos los prohibidos minimales de $[h, 2,1]$, con $h \geq 3$, que son VPT pueden ser obtenidos de un grafo $(h+1)$-crítico.

Notar que, tomando $h=3$, obtenemos una caracterización por subgrafos inducidos prohibidos minimales para la clase VPT $\cap$ EPT $=\mathrm{EPT}$ $\cap$ Cordal $=[3,2,2]=[3,2,1](\operatorname{ver}[15])$.

Nos gustaría encontrar algunas propiedades sobre la representación de los grafos VPT que son minimales no- $[h, 2,1]$, para $h \geq 3$.

- En el Capítulo 5 damos una nueva familia finita de subgrafos inducidos prohibidos minimales para la clase EPT, pero sabemos que esta familia es incompleta pues por ejemplo el grafo $A_{4}$ (ver Figura 7.2) es un grafo minimal no-EPT [19] y $A_{4}$ no está en nuestra familia de subgrafos inducidos prohibidos minimales.

Estamos trabajando en encontrar nuevos subgrafos inducidos prohibidos minimales para la clase EPT. 


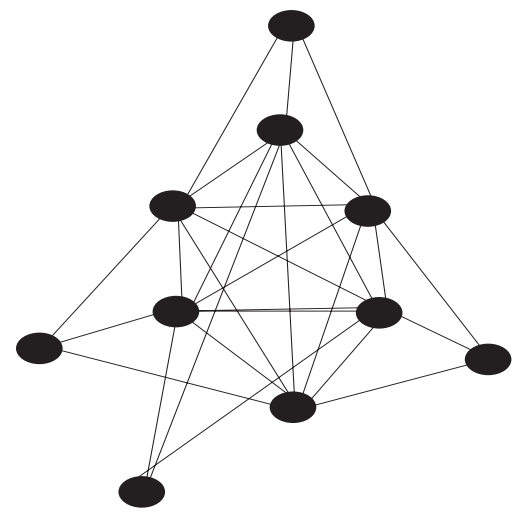

Figura 7.2: $A_{4}$ no es un grafo EPT.

- En [19], Golumbic, Lipshteyn y Stern estudiaron varios aspectos de las clases $[h, 2,2]$, para $h \geq 2$. En particular probaron que en la clase de los grafos EPT, la recíproca del Teorema 6.1 es verdadera para $h=3$ y es falsa para $h=4$. Usaron varios resultados generales de las clases de los grafos Cordales y Débilmente Cordales (ver [7] para las definiciones) los cuales se aplican sólo cuando $h=3$ o $h=4$. Dejaron abierta la pregunta de saber si la recíproca del Teorema 6.1 es verdadera para $h \geq 5$. En el Capítulo 6, respondemos negativamente a esta pregunta. Describimos, para todo $h \geq 5$, un grafo EPT que es libre de $\left\{C_{n} \mid n>h\right\}$ y, sin embargo, no puede ser representado en un árbol huésped de grado $h$.

Estamos trabajando en encontrar la lista completa de grafos EPT que son subgrafos inducidos prohibidos minimales para la clase $[h, 2,2]$. 


\section{Bibliografía}

[1] L. Alcón, M. Gutierrez, M. P. Mazzoleni, A necessary condition for EPT graphs and a new family of minimal forbidden subgraphs, Matemática Contemporánea. 39 (2010) 111-120.

[2] L. Alcón, M. Gutierrez, M. P. Mazzoleni, EPT graphs on bounded degree trees, aceptado para publicar en Matemática Contemporánea. (2014)

[3] L. Alcón, M. Gutierrez, M. P. Mazzoleni, Recognizing vertex intersection graphs of paths on bounded degree trees, Discrete Applied Mathematics. 162 (2014) 70-77.

[4] L. Alcón, M. Gutierrez, M. P. Mazzoleni, On minimal non [h,2,1] graphs, Electronics Notes in Discrete Mathematics. 44 (2013) 115-120.

[5] L. Alcón, M. Gutierrez, M. P. Mazzoleni, Characterizing [h,2,1] graphs by minimal forbidden induced subgraphs, arXiv:1307.2139, manuscript. (2013).

[6] J. Bondy, U. Murty, Graph Theory, Springer, New York, NY, USA. (2007). 
[7] A. Brandstädt, V. B. Le, J. P. Spinrad, Graph Classes: A Survey, SIAM Monographs on Discrete Mathematics and Applications. (1999).

[8] C. Berge, Graphs and Hypergraphs, North-Holland, Amsterdam. (1973).

[9] M. R. Cerioli, H. I. Nobrega, P. Viana, A partial characterization by forbidden subgraphs of edge path graphs, Cologne-Twente Workshop on Graphs and Combinatorial Optimization. (2011) 109-112.

[10] M. Chudnovsky, N. Robertson, P. Seymour, R. Thomas, The strong perfect graph theorem, Annals of Mathematics. 164 (2006) 51-229.

[11] E. Cohen, M. C. Golumbic, M. Lipshteyn, M. Stern, What is between chordal and weakly chordal graphs?, Graph-Theoretic Concepts in Computer Science. Lecture Notes in Computer Science. 5344 (2008) 275-286.

[12] N. Eaton, Z. Füredi, A. V. Kostochka, J. Skokan, Tree representations of graphs, Europen Journal of Combinatorics. 28 (2007) 1087-1098.

[13] F. Gavril, The intersection graphs of subtrees in a tree are exactly the chordal graphs, Journal of Combinatorial Theory. 16 (1974) 47-56.

[14] F. Gavril, A recognition algorithm for the intersection graphs of paths in trees, Discrete Math. 23 (1978) 211-227.

[15] M. C. Golumbic, R. E. Jamison, Edge and vertex intersection of paths in a tree, Discrete Mathematics. 38 (1985) 151-159.

[16] M. C. Golumbic, R. E. Jamison The edge intersection graphs of paths in a tree, Journal of Combinatorial Theory. 55 (1985) 8-22. 
[17] M. C. Golumbic, Algorithmic Graph Theory and Perfect Graphs, In: Annals of Discrete Mathematics, North Holland. 57 (2004).

[18] M. C. Golumbic, M. Lipshteyn, M. Stern, The k-edge intersection graphs of paths in a tree, Discrete Applied Mathematics. 156 (2008) 451-461.

[19] M. C. Golumbic, M. Lipshteyn, M. Stern, Equivalences and the complete hierarchy of intersection graphs of paths in a tree, Discrete Applied Mathematics. 156 (2008) 3203-3215.

[20] M. C. Golumbic, M. Lipshteyn, M. Stern, Representing edge intersection graphs of paths on degree 4 trees, Discrete Mathematics. 308 (2008) 1381-1387.

[21] M. C. Golumbic, M. Lipshteyn, M. Stern, Intersection models of weakly chordal graphs, Discrete Applied Mathematics. 157 (2009) 2031-2047.

[22] D. L. Greenwell, R. L. Hemminger, J. B. Klerlein Forbidden subgraphs, In: F. Hoffman, R. Lavow and R. Thomas (Eds.), Proceedings of the Fourth Southeastern Conference on Combinatorics, Graph Theory, and Computing. Utilitas Mathematica. (1973) 389-394.

[23] M. Grötschel, L. Lovász, A. Schrijver, The ellipsoid method and its consequences in combinatorial optimization, Combinatorica 1. (1981) 169-197.

[24] M. Gutierrez, S. B. Tondato, J. L. Szwarcfiter, A forbidden subgraph characterization of paths graphs, Electronics Notes in Discrete Mathematics. Proceedings of GRACO 2005. 19 (2005) 281-287. 
[25] R. E. Jamison, H. M. Mulder, Tolerance intersection graphs on binary trees with constant tolerance 3, Discrete Mathematics. 215 (2000) 115131.

[26] R. E. Jamison, H. M. Mulder, Constant tolerance intersection graphs of subtress of a tree, Discrete Mathematics. 290 (2005) 27-46.

[27] R. Karp, Reducibility among combinatorial problems, In: R. E. Miller and J. W. Thatcher, editors, Complexity of Computer Computations. (1972) 85-104.

[28] C. G. Lekkerkerker, J. C. Boland, Representation of a finite graph by a set of intervals on the real line, Fundamenta Mathematicae. 51 (1962) 45-64.

[29] B. Lévêque, F. Maffray, M. Preissmann, Characterizing path graphs by forbidden induced subgraphs, Journal of Graph Theory 62 (2009) 369384.

[30] T. McKee, F. McMorris, Topics in Intersection Graph Theory, SIAM: Philadelphia, PA, USA. (1999).

[31] C. L. Monma, V. K. Wei, Intersection graphs of paths in a tree, Journal of Combinatorial Theory. (1986) 140-181.

[32] F. R. Morris, E. Scheinerman, Connectivity threshold for random chordal graphs, Graphs and Combinatorics. 7 (1991) 177-181.

[33] A. A. Schaffer, A faster algorithm to recognize undirected path graphs, Discrete Applied Mathematics. 43 (1993) 261-295. 
[34] M. M. Syslo, On characterizations of cycle graphs and on other families of intersection graphs, Tech. Rep., Institute of Computer Science, University of Wroclaw, Poland. 40 (1978).

[35] M. M. Syslo, Triangulated edge intersection graphs of paths in a tree, Discrete Mathematics. 55 (1985) 217-220.

[36] R. Tarjan, Descomposition by clique separators, Discrete Mathematics. 55 (1985) 221-232. 